\title{
18. ORGANOFACIES VARIATIONS IN SEDIMENTS FROM THE CONTINENTAL SLOPE AND RISE OF THE NEW JERSEY CONTINENTAL MARGIN (SITES 903 AND 905) ${ }^{1}$
}

\author{
Jan Hendrik van der Smissen ${ }^{2}$ and Jürgen Rullkötter ${ }^{2}$
}

\begin{abstract}
This study describes molecular organic geochemical investigations of 14 samples from Site 903 off New Jersey, as well as bulk analysis of a great number of sediments from Sites 903 and 905 . Detailed lipid analysis of the solvent-extractable organic matter exhibits a complex assemblage of marine, terrigenous, and bacterial compounds. $n$-Alkanes and $n$-alcohols are the most abundant compounds in extracts of sediments from Site 903, followed by steroidal alcohols in considerably smaller concentrations that decrease with depth. Analysis of long-chain unsaturated ketones for reconstruction of paleo-sea surface temperature results in an average water temperature estimate of $25^{\circ} \mathrm{C}$ since the middle Miocene. The results indicate continuous supply of terrigenous organic material to the region with occasional conditions of better marine organic matter preservation, particularly in Oligocene to middle Miocene times. Some variations in organic matter composition may be caused by sea-level changes. A warm current analogous to the present Gulf Stream appears to have existed off New Jersey at least since the Oligocene.
\end{abstract}

\section{INTRODUCTION}

Sediments deposited along ancient continental margins represent a significant portion of the geological record and comprise a sensitive and long-term record of environmental change. During regression phases of the ocean caused by climatic change, major parts of the continental shelf area fell dry (Mountain and Miller, 1993/94). At that time, rivers transported terrestrial material to the outer continental shelf and slope. Cyclic variations in the supply of terrigenous organic matter over geological times should be documented by the composition of inherited fossil organic matter, so that these sediments offer a possibility for organic geochemical investigations of environmental change.

Accumulation and preservation of marine organic matter are controlled by a number of different factors, for example, the primary biological productivity in the surface water, the supply of terrigenous organic material from the continent (which in turn is a function of continental climate), the amount of oxygen in the water column, and microbial and chemical diagenetic alteration in the sediments. The continental rise off New Jersey, at least in modern times, is influenced by the Western Boundary Undercurrent (WBU), a strong southwest-flowing bottom current composed primarily of North Atlantic Deep Water with an admixture of Antarctic Bottom Water (Amos et al., 1971; Heezen et al., 1966; McCave and Tucholke, 1986). This well mixed and oxygen-rich water mass affects the accumulation of labile organic material, so that only a small part of the original marine organic matter and terrigenous supply will be preserved, but this effect may have varied with time. Thus, an investigation of the quantity and composition of organic matter in deep-sea sediments on the New Jersey continental margin may still provide information on changes in paleoenvironment, such as sea-level changes and associated terrigenous supply by river transport.

Molecular organic geochemical studies of sediments are based mostly upon the presence of chemical fossils (or biological markers),

Mountain, G.S., Miller, K.G., Blum, P., Poag, C.W., and Twichell, D.C. (Eds.), 1996. Proc. ODP, Sci. Results, 150: College Station, TX (Ocean Drilling Program).

2Institute of Chemistry and Biology of the Marine Environment (ICBM), Carl von Ossietzky Universität Oldenburg, P.O. Box 2503, D-26111 Oldenburg, Federal Republic of Germany. a group of compounds that, from their occurrence in the organic matter fraction of a sediment, provides a link to a specific type of organism or a larger family of organisms (Eglinton and Calvin, 1967; Philp, 1985; Poynter and Eglinton, 1990). Examples of such compounds are $4 \alpha, 23,24$-trimethyl- $5 \alpha(\mathrm{H})$-cholest-22-en-3 $\beta$-ol (dinosterol) or 24-ethylcholest-5-en-3 $\beta$-ol as indicators of dinoflagellates or higher land plants, respectively (e.g., Volkman, 1986). Thus, in a more specific way than bulk organic geochemical parameters, biological marker assemblages reflect paleoenvironmental changes of organic matter deposition.

The coastal plain, shelf, and slope off New Jersey have long been recognized as a source of information on sea-level history. They provide a record of transgressive and regressive sequences that can be correlated with other proxies of sea-level changes (Owens and Gohn, 1985; Olsson et al.,1987; Olsson and Wise, 1987). For a selected set of samples from Sites 903 and 905, this study reports results of an investigation of bulk characteristics (i.e., total organic carbon, carbonate, and sulfur contents) and of the composition of extractable lipids covering a range of compound classes (hydrocarbons, sterols, fatty acids, and alkenones), using gas chromatography (GC) and combined gas chromatography-mass spectrometry (GC/MS), as a first attempt to relate the results to climatic changes and sea-level variations.

\section{LITHOLOGICAL DESCRIPTION AND ANALYTICAL METHODS}

\section{Lithology}

Ocean Drilling Program (ODP) Site 903 (Fig. 1) is located at $38^{\circ} 56.298^{\prime} \mathrm{N}$ and $72^{\circ} 49.032^{\prime} \mathrm{W}$ at the distal end of the continental shelf (upper continental slope; water depth 446 meters [m]; Shipboard Scientific Party, 1994a). Drilling at Site 903 recovered 1149.7 $\mathrm{m}$ of sediment spanning a record from the middle Eocene to the Pleistocene (the last $45 \mathrm{Ma}$ ). The sediments consist of Pleistocene to Pliocene silty clays and sand (lithologic Units I and II); upper and middle Miocene alternations of silty clays and glauconitic sands (Unit III); middle Miocene diatomaceous silty clays to clayey silts including slumps and debris flow deposits (Unit IV); lower to middle Miocene glauconitic, diatomaceous, organic-matter-rich silty claystones (Unit V); lowermost Miocene to upper Oligocene silty claystones (Unit VI); and upper Eocene clayey nannofossil chalks (Unit VII). 


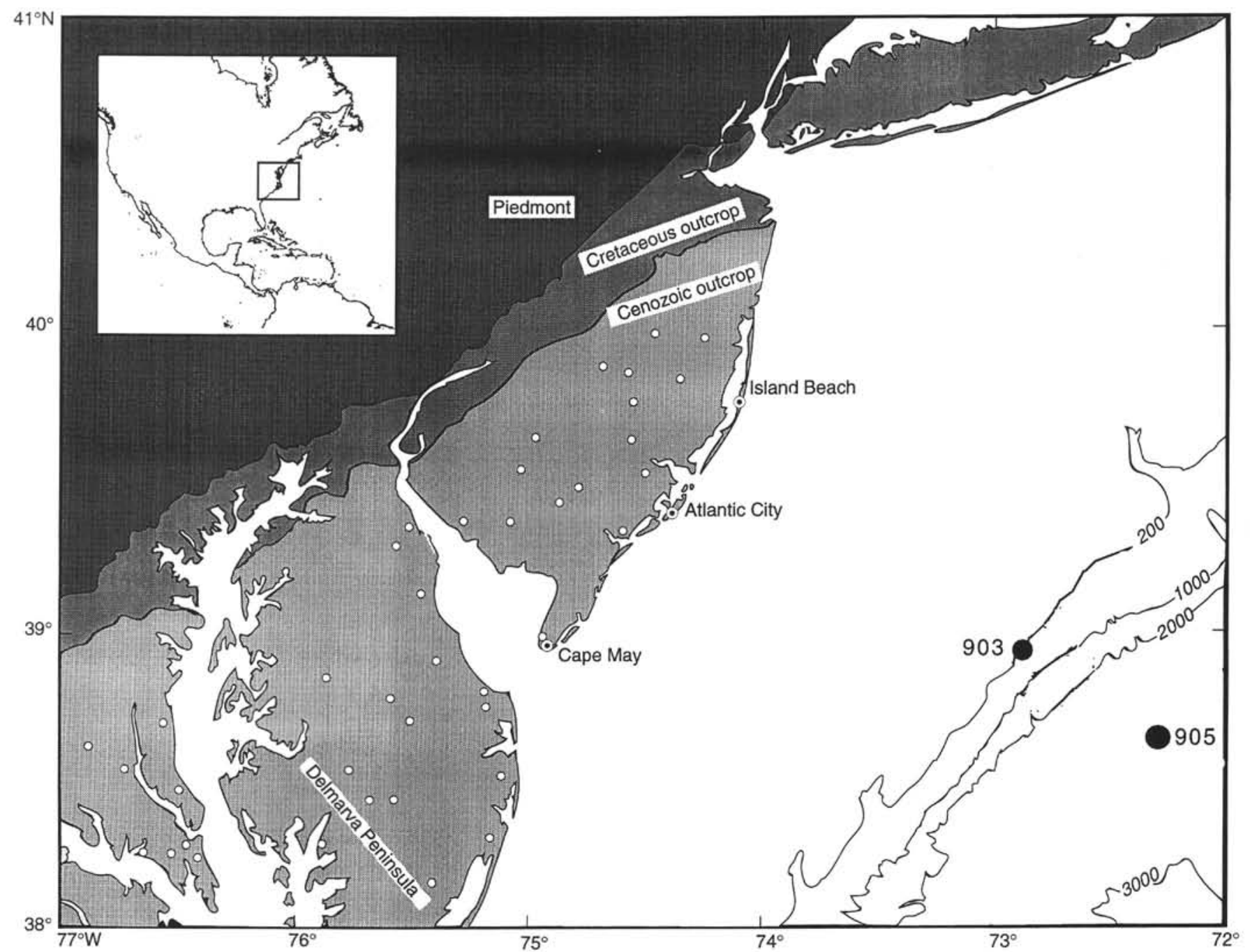

Figure 1. Drilling locations on the New Jersey continental margin during Leg 150.

Drilling was also performed at Site $905\left(38^{\circ} 36.828^{\prime} \mathrm{N}, 72^{\circ}\right.$ $17.024^{\prime}$ W; water depth 2698 m; Shipboard Scientific Party, 1994b), which is located at the continental rise off New Jersey (Fig. 1). Four lithostratigraphic units were recognized at Site 905 down to a terminal depth of $910.6 \mathrm{mbsf}$. They comprise lower Pleistocene conglomeratic clay almost entirely representing mass-transport deposits of terrigenous origin (Unit I), lower Pleistocene to upper Miocene homogeneous silty clay with low terrigenous contribution (Unit II), several complex upper to middle Miocene units of conglomeratic clay (Unit III), and upper middle Miocene silty clay with little terrigenous influence and abundant diatoms (Unit IV).

\section{Analytical Methods}

A total of 122 samples (received frozen) from ODP Sites 903 and 905 were analyzed for bulk geochemical characteristics. Aliquots of the freeze-dried and ground sediments were used to determine total carbon (TC), total organic carbon (TOC), and total sulfur (TS) contents with a LECO SC-444 instrument. Before TOC determination, the samples were treated with hydrochloric acid to remove carbonates. Carbonate contents were calculated by difference and expressed as percent calcium carbonate. The release of hydrocarbons and carbon dioxide during temperature-programmed pyrolysis was deter- mined as hydrogen indices (HI: $\mathrm{mg} \mathrm{HC} / \mathrm{g}$ TOC) and oxygen indices (OI: $\mathrm{mg} \mathrm{CO}_{2} / \mathrm{g}$ TOC), respectively (Espitalié et al., 1977).

From the total number of samples, 14 were selected for detailed studies of the molecular organic matter composition. The ground sediments were ultrasonically extracted three times ( $15 \mathrm{~min}$ each) with dichlormethane (containing 1\% methanol). After each step, the solvent was decanted. All three extracts were combined. Known quantities of internal standards (squalane, erucic acid, $5 \alpha(\mathrm{H})$-androstan-17one) were added to allow later quantitative measurement of lipid abundances. Before separation into molecular group fractions, the $n$ hexane soluble compounds (after precipitation of asphaltenes) were divided into two aliquots. The larger portion $(80 \%)$ was separated by medium-pressure liquid chromatography (MPLC) (Radke et al., 1980 ) into compound classes of different polarities, that is, aliphatic hydrocarbons, aromatic hydrocarbons, and polar hetero(NSO)-components. Carboxylic acids were separated from the polar compounds by using a column filled with silica gel 60 impregnated with $0.5 \mathrm{~g}$ $\mathrm{KOH}$ dissolved in $10 \mathrm{~mL}$ iso-propanol. The nonacidic compounds were eluted from the column with dichlormethane. Alcohols and ketones were separated from the acid-free NSO fraction by flash chromatography (Still et al., 1978) under $\mathrm{N}_{2}$ pressure using dichloromethane and dichloromethane $/ 10 \%$ methanol as eluents. Where necessary, the extract fractions were derivatized with diazomethane 
and N-methyl-N-trimethylsilyltrifluoroacetamide (MSTFA) before analysis.

GC analysis was conducted on a Hewlett-Packard 5890 series II instrument equipped with a temperature-programmable injector system (Gerstel KAS 3), a fused silica capillary column ( $30 \mathrm{~m} \times 0.25$ $\mathrm{mm}$ i.d., film thickness $0.25 \mu \mathrm{m}$ ) and a flame ionization detector (FID). The temperature of the GC oven was programmed from $60^{\circ} \mathrm{C}$ (1 min isothermal) to $300^{\circ} \mathrm{C}(50 \mathrm{~min}$ isothermal $)$ at $3^{\circ} \mathrm{C} / \mathrm{min}$. The injector temperature was programmed from $60^{\circ} \mathrm{C}(5 \mathrm{~s}$ hold time $)$ to $300^{\circ} \mathrm{C}\left(60 \mathrm{~s}\right.$ hold time) at $8^{\circ} \mathrm{C} / \mathrm{s}$. GC/MS measurements were performed with the same type of GC system, under the conditions described above, coupled to a Finnigan SSQ 710 B mass spectrometer operated at $70 \mathrm{eV}$ with a cyclic scan time of $1 \mathrm{scan} / \mathrm{s}$. Compounds were identified by comparing their relative gas chromatographic retention times and mass spectra with those reported in the literature. Quantification was performed relative to the amount of internal standards in the gas chromatograms.

\section{RESULTS AND DISCUSSION Bulk Parameters}

The downhole variations in bulk composition of the sediments from Sites 903 and 905 in terms of organic carbon, sulfur, and carbonate contents are shown in Figures 2 and 3. For better correlations, curve fits were calculated using a five-point weighted running average smoothing function. Data of organic carbon, $\mathrm{CaCO}_{3}$, and elemental analysis of sediments at Site 903 and 905 are listed in Tables 1 and 2. Rock-Eval pyrolysis, and extract yields of selected samples from Site 903 are summarized in Table 3. Eocene sediments at Site 903 are rich in carbonate (35\% to nearly $60 \%$ ), but lean in organic carbon (around $0.5 \%$ ). Carbonate concentrations decrease significantly in the Oligocene and are very low throughout most of the Miocene and Pliocene, with slightly higher values on average in the continental rise sediments compared to those from the upper slope. Carbonate concentrations of about $10 \%$ are typical of the Pleistocene sections at both sites.

The decrease in carbonate content in the Oligocene at Site 903 is accompanied by a strong increase in organic carbon concentration. Similarly high TOC values of more than $2 \%$ were measured for the lower Miocene and part of the middle Miocene sections at Site 903 whereas in the shallower part of the middle Miocene, the upper Miocene, and the Pliocene sections at both sites TOC values vary between $1 \%$ and $1.5 \%$ for most of the samples analyzed. The variations of organic matter concentrations are probably not simply related to dilution with biogenic carbonate, because there is no general correlation between carbonate and organic carbon contents over a larger depth range. Even from Eocene to Oligocene, where there is an obvious negative correlation between the two carbon parameters, changes in organic matter quality (e.g., hydrogen indices; Table 3 ) are evidence against simple dilution. The Pleistocene sediments are characterized by low organic carbon contents $(<0.5 \%)$, with a few exceptions at the continental rise, which probably represent slump clasts of more organic-matter-rich (Miocene?) sediment originally deposited at a shallower site under conditions of better preservation of organic matter.

Microbial sulfate reduction leading to relatively high total sulfur contents $(1 \%-2 \%)$ in the sediment was most effective in those sections of the sedimentary column that also have preserved higher amounts of organic carbon. This indicates that the deposition of labile organic matter (probably marine to a large extent) was higher in Oligocene to Pliocene times than it was in the Eocene and the Pleistocene. Of the organic material preserved in the sediments, the marine organic matter portion appears highest during the lower and middle Miocene, according to the hydrogen index values from RockEval pyrolysis (Table 3), but there is probably still a significant admixture of terrigenous organic matter because absolute $\mathrm{HI}$ values are still below $300 \mathrm{mg} \mathrm{HC} / \mathrm{g}$ TOC. High concentrations of methane in these sediments suggest that pronounced methanogenesis has metabolized a significant part of the deposited labile marine organic matter. Eocene as well as upper Miocene and younger sediments, according to their $\mathrm{HI}$ and $\mathrm{OI}$ values, mostly contain terrigenous and oxidized residual organic matter; oxidation may partly be the result of strong microbial reworking near the sediment/water interface. The Rock-Eval data fit well into the HI profile reported by the shipboard party (Shipboard Scientific Party, 1994a, 1994b).

The amount of extractable organic material varies between 10 and $20 \mathrm{mg} / \mathrm{g}$ TOC; thus, they are in typical range of deep-sea sediments containing immature organic matter. The total extracts are dominated by the NSO fractions, followed by asphaltenes, and the aliphatic and aromatic hydrocarbons (Fig. 4). All core samples selected for molecular analysis contain a significant proportion of extractable elemental sulfur $(10 \%-30 \%$ of total extract).

\section{Molecular Investigations}

\section{Aliphatic Hydrocarbons}

The most important biogenic constituents in the aliphatic hydrocarbon fractions are straight-chain $n$-alkanes, of which the $\mathrm{C}_{29}$ or $\mathrm{C}_{31}$ members are predominant. The $n$-alkane distribution patterns are similar for most of the samples studied. Carbon preference index values $\left(\mathrm{CPI}_{31}\right)$ (Bray and Evans, 1961) vary between 2.8 and 5.8, suggesting that there was a significant supply of terrigenous lipids from epicuticular waxes of higher land plants to the sediments (Eglinton and Calvin, 1967; Kolattukudy, 1976). A typical example of a Pleistocene sediment with only a minor proportion of marine organic matter is shown in Figure 5A. Steroid and hopanoid hydrocarbons, irrespective of progress in diagenesis, are minor compounds when compared with $n$-alkanes in these samples. In contrast to this, middle and lower Miocene sediments in addition to $n$-alkanes contain complex mixtures of saturated and unsaturated steroid and hopanoid hydrocarbons (Fig. 5B). Absolute concentrations and $n$-alkane carbon number distributions of all 14 sediments used for molecular analysis are shown in Figure 6. The $n$-alkane distribution of Sample 150-903A$13 \mathrm{H}-4,87-92 \mathrm{~cm}$ (119.37 mbsf, Pleistocene) indicates contamination as evident from the abundance of lower-molecular-weight homologs, the low CPI value, and the high absolute concentrations. Apart from that, $n$-alkane concentrations (normalized to total organic carbon) appear to increase with depth down to the upper part of the middle Miocene section (about $600 \mathrm{mbsf}$ ) and then to decrease toward the bottom of the hole. This is consistent with the better conditions for organic matter preservation in middle Miocene to Oligocene sediments which may also have affected the accumulation rate of terrigenous $n$ alkanes.

Major biomarker compounds identified in the sediments enriched in marine organic matter (e.g., Fig. 5B) are sterenes in the carbon number range from $C_{27}$ to $C_{29}$ (mainly $\Delta^{4}$ and $\Delta^{5}$ isomers), which are likely to be mainly of marine planktonic origin although a contribution from terrestrial higher plants cannot be fully excluded (Volkman, 1986). In agreement with the suggested source of the $n$-alkanes, however, are the degraded triterpenoids of the des-A-oleanene and -ursene types, which are specific for land-derived angiosperm organic matter. Among the hopanoid compounds of bacterial origin, hop-17(21)-enes are the major constituents whereas $17 \beta(\mathrm{H}), 21 \beta(\mathrm{H})$-hopanes are present in smaller concentrations. In more deeply buried samples, fern-8-ene and fern-9(11)-ene, the more stable isomers of this group of compounds, are present in small amounts. All these compounds are typical of bacterial origin (e.g., Brassell, 1985; McEvoy et al., 1981; Volkman et al., 1986).

\section{n-Alcohols and Sterols}

A typical partial reconstructed ion chromatogram from GC/MS analysis of the alcohol fractions (analyzed as trimethylsilyl ethers) of 


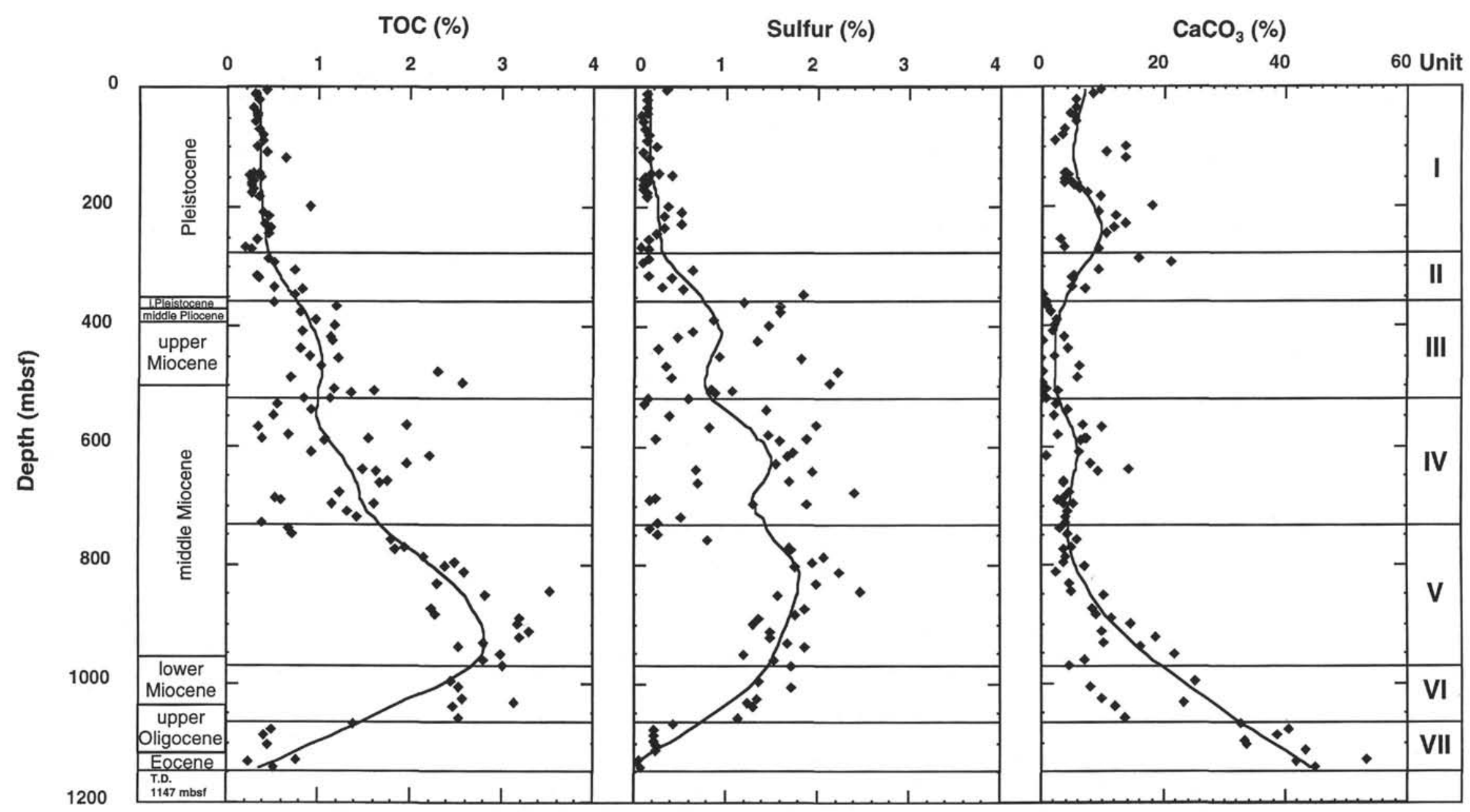

Figure 2. Depth profiles of total organic carbon, sulfur, and carbonate contents in sediments from the upper continental slope off New Jersey, Site 903 . Curves were calculated by a five-point weighted running average smoothing function. 


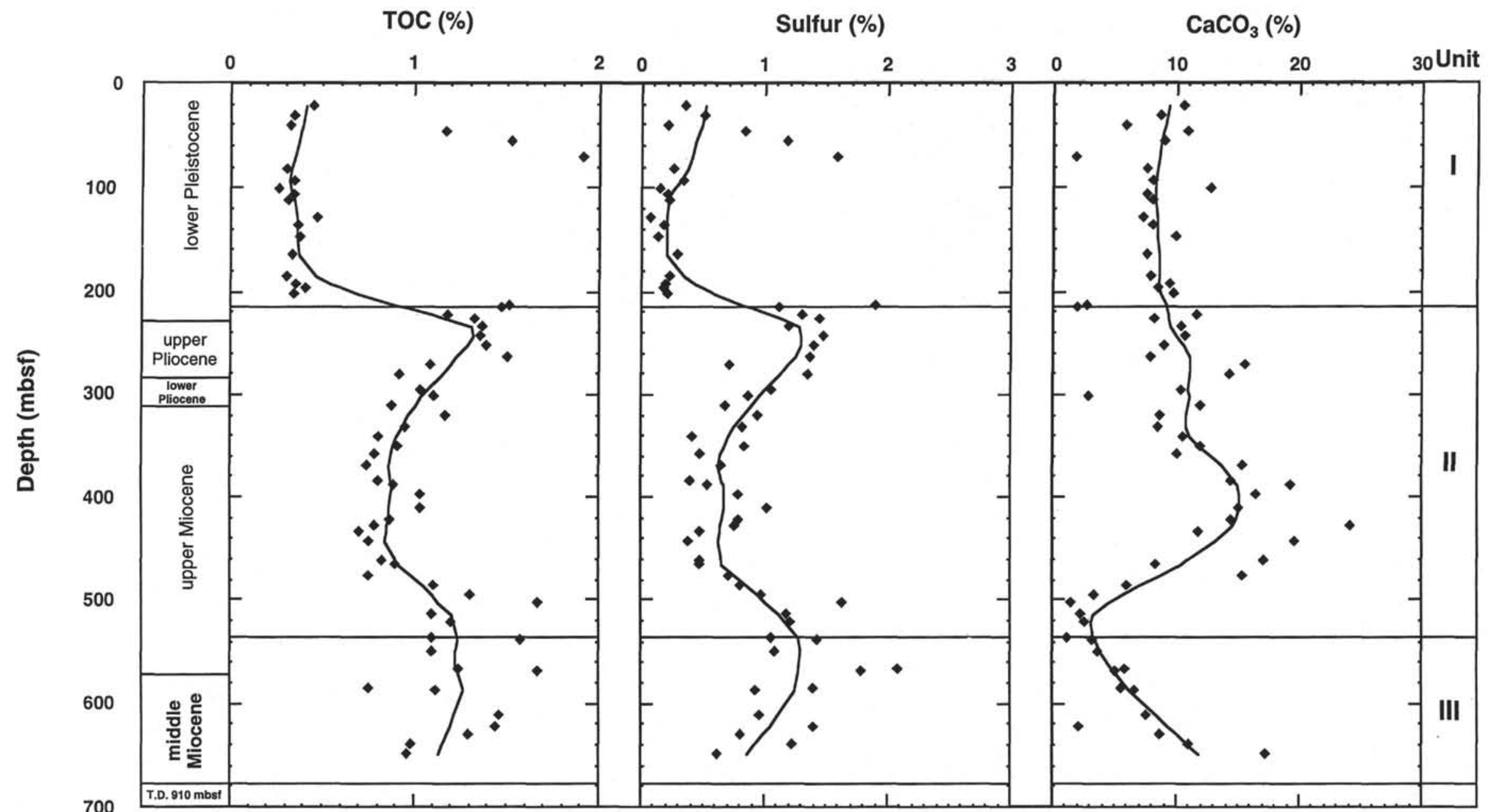

Figure 3. Depth profiles of total organic carbon, sulfur, and carbonate contents in sediments from the continental rise off New Jersey, Site 905 . Curves were calculated by a five-point weighted running average smoothing function. 
Table 1. Lithology, organic carbon content, and elemental analysis data for core samples from Site 903.

\begin{tabular}{|c|c|c|c|c|c|}
\hline $\begin{array}{l}\text { Core, section, } \\
\text { interval }(\mathrm{cm})\end{array}$ & $\begin{array}{l}\text { Depth } \\
\text { (mbsf) }\end{array}$ & Lithology & $\begin{array}{l}\text { TOC } \\
\text { (wt\%) }\end{array}$ & $\begin{array}{l}\mathrm{CaCO}_{3} \\
\text { (wt\%) }\end{array}$ & $\begin{array}{l}\text { Sulfur } \\
\text { (wt\%) }\end{array}$ \\
\hline \multicolumn{6}{|l|}{$150-903 \mathrm{~A}-$} \\
\hline $1 \mathrm{H}-4,94-100$ & 5.4 & Homogeneous clayey silt & 0.44 & 9.7 & 0.36 \\
\hline $2 \mathrm{H}-3,80-86$ & 13.3 & Clayey silt with fine sand layers & 0.32 & 8.5 & 0.15 \\
\hline $3 \mathrm{H}-3,80-86$ & 22.8 & Heavily bioturbated clayey silt & 0.35 & 5.5 & 0.14 \\
\hline $4 \mathrm{H}-5,76-82$ & 35.2 & Heavily bioturbated clayey silt and silty clay & 0.30 & 5.5 & 0.13 \\
\hline $5 H-5,81-86$ & 44.8 & Gray clayey silt and silty clay, heavily bioturbated & 0.33 & 4.6 & 0.14 \\
\hline $6 \mathrm{H}-2,62-68$ & 49.6 & Silty clay with sand- and black sulfide-filled burrows & 0.33 & 5.4 & 0.09 \\
\hline $7 \mathrm{H}-2,65-71$ & 59.1 & Heavily bioturbated silty clay and very fine sand & 0.31 & 5.7 & 0.11 \\
\hline $8 \mathrm{H}-3,93-99$ & 70.4 & Silty clay and clayey silt & 0.35 & 3.7 & 0.12 \\
\hline $9 \mathrm{H}-4,5-11$ & 80.5 & Silty clay and clayey silt & 0.40 & 3.3 & 0.17 \\
\hline $10 \mathrm{H}-3,90-95$ & 89.4 & Heavily bioturbated silty clay and clayey silt & 0.40 & 2.1 & 0.15 \\
\hline $11 \mathrm{H}-4,6-12$ & 99.5 & Silty clay and clayey silt with pyritic very fine to fine sand & 0.33 & 13.9 & 0.25 \\
\hline $12 \mathrm{H}-3,83-90$ & 108.3 & Silty clay and clayey silt; pyritic, very dark sand fills burrows & 0.43 & 10.5 & 0.11 \\
\hline $13 \mathrm{H}-4,87-92$ & 119.4 & Silty clay and clayey silt & 0.65 & 13.9 & 0.16 \\
\hline $16 \mathrm{H}-2,52-57$ & 145.0 & Slightly bioturbated silty clay and clayey silt & 0.35 & 4.2 & 0.27 \\
\hline $16 \mathrm{H}-2,100-103$ & 146.0 & Silty clay and clayey silt & 0.30 & 3.8 & 0.19 \\
\hline $17 \mathrm{H}-1,20-23$ & 149.2 & Silty clay and clayey silt with iron sulfide-rich black fine sand fills burrows & 0.26 & 4.3 & 0.41 \\
\hline $17 \mathrm{H}-2,68-74$ & 151.2 & Silty clay and clayey silt & 0.37 & 4.2 & 0.13 \\
\hline $18 \mathrm{H}-1,20-25$ & 154.2 & Slightly bioturbated silty clay and clayey silt & 0.28 & 3.6 & 0.10 \\
\hline $18 \mathrm{H}-2,89-91$ & 156.4 & Slightly bioturbated silty clay and clayey silt & 0.29 & 4.7 & 0.14 \\
\hline $19 \mathrm{H}-2,90-96$ & 162.4 & Slightly to moderately bioturbated silty clay and clayey silt & 0.28 & 3.7 & 0.14 \\
\hline $20 \mathrm{H}-1,90-96$ & 165.4 & Slightly bioturbated silty clay and clayey silt & 0.27 & 5.3 & 0.11 \\
\hline $21 X-1,90-96$ & 170.4 & Bioturbated silty clay & 0.29 & 6.3 & 0.10 \\
\hline $22 \mathrm{X}-3,60-66$ & 176.4 & Moderately bioturbated clayey silt & 0.28 & 7.6 & 0.15 \\
\hline $23 \mathrm{X}-1,137-143$ & 183.7 & Moderately bioturbated clayey silt & 0.35 & 9.6 & 0.14 \\
\hline $24 X-2,24-29$ & 198.8 & Homogeneous silty clay and diatomaceous clay & 0.91 & 18.0 & 0.37 \\
\hline $25 \mathrm{X}-6,12-18$ & 209.0 & Silty clay and sandy silt & 0.39 & 9.4 & 0.53 \\
\hline $26 \mathrm{X}-3,83-88$ & 214.8 & Homogeneous green diatomaceous silty clay & 0.45 & 12.3 & 0.34 \\
\hline $27 X-5,120-126$ & 227.8 & Silty clay and sandy silty clay & 0.42 & 13.8 & 0.53 \\
\hline $28 X-4,99-105$ & 235.8 & Variably bioturbated clayey silt & 0.48 & 11.8 & 0.34 \\
\hline $29 \mathrm{X}-3,90-95$ & 243.9 & Slightly to moderately bioturbated sandy silty clay and clayey silt & 0.45 & 10.6 & 0.25 \\
\hline $30 \times-3,57-63$ & 253.3 & Heavily bioturbated silty clay and sand/silt/clay & 0.33 & 3.0 & 0.16 \\
\hline $31 X-5,20-25$ & 265.5 & Slightly to moderately bioturbated sand-silt-clay & 0.21 & 3.8 & 0.08 \\
\hline $32 \mathrm{X}-1,16-20$ & 269.0 & Silty clay and sandy silty clay & 0.27 & 9.5 & 0.17 \\
\hline $33 X-5,60-65$ & 284.8 & Slightly bioturbated silty clay and sandy silty clay & 0.45 & 15.9 & 0.16 \\
\hline $34 X-5,80-86$ & 294.0 & Silty clay and clayey silt & 0.53 & 21.3 & 0.10 \\
\hline $35 X-6,10-16$ & 305.5 & Silty sand & 0.76 & 9.5 & 0.65 \\
\hline $36 X-3,17-20$ & 316.6 & Slightly bioturbated silty clay & 0.33 & 5.4 & 0.16 \\
\hline $37 \mathrm{X}-2,17-19$ & 317.9 & Greenish gray silty clay & 0.35 & 5.0 & 0.41 \\
\hline $38 X-6,17-20$ & 334.4 & Homogenous silty clay & 0.53 & 4.9 & 0.31 \\
\hline $39 X-1,127-133$ & 337.7 & Silty clay, homogeneous sandy clay and fine to medium sand & 0.84 & 7.2 & 0.54 \\
\hline $40 X-2,85-91$ & 348.3 & Glauconitic sand and silty sand & 0.76 & 0.2 & 1.85 \\
\hline $41 X-4,52-58$ & 360.7 & Glauconitic sand, sandy silt and clayey silt & 0.53 & 0.8 & 1.20 \\
\hline $42 X-2,51-56$ & 367.4 & Sandy silt and homogeneous clayey silt & 1.21 & 1.0 & 1.60 \\
\hline $43 \mathrm{X}-2,53-59$ & 376.9 & Moderately bioturbated sandy silt & 0.81 & 1.7 & 1.60 \\
\hline $44 X-4,45-51$ & 389.5 & Silty sand and silty clay & 0.97 & 2.5 & 0.88 \\
\hline $45 X-3,60-66$ & 397.9 & Heavily bioturbated silty clay & 1.19 & 2.1 & 1.48 \\
\hline $46 X-4,101-106$ & 409.0 & Moderately to heavily bioturbated clayey silt, silt and sandy silt & 0.84 & 1.8 & 0.64 \\
\hline $47 X-4,30-36$ & 418.5 & Silty clay, glauconitic sandy silt and clay & 1.14 & 3.6 & 0.48 \\
\hline $48 X-2,60-66$ & 425.4 & Silty clay and glauconitic sandy silt & 1.16 & 0.3 & 1.36 \\
\hline $49 X-4,33-38$ & 437.8 & Silty clay and glauconitic sandy silt & 0.81 & 4.5 & 0.28 \\
\hline $50 X-5,44-50$ & 449.1 & Heavily bioturbated glauconitic silty sand & 0.91 & 2.1 & 0.94 \\
\hline $51 X-2,40-46$ & 454.1 & Heavily bioturbated glauconitic silty sand and clay & 1.23 & 0.1 & 1.83 \\
\hline $52 \times-3,54-60$ & 465.4 & Moderately bioturbated silty clay and sandy clay & 1.04 & 6.1 & 0.36 \\
\hline $53 X-4,90-96$ & 477.0 & Slightly bioturbated silty clay and sandy silty clay & 2.31 & 0.3 & 2.22 \\
\hline $54 X-4,40-46$ & 486.2 & Weakly bioturbated silty clay and sandy clayey silt & 0.71 & 6.0 & 0.41 \\
\hline $55 \times-4,40-46$ & 495.8 & Silty clay and sandy, clayey silt & 2.58 & 0.2 & 2.14 \\
\hline $56 X-4,90-93$ & 506.0 & Moderately bioturbated sandy, silty clay & 1.19 & 0.9 & 0.86 \\
\hline $57 X-1,56-59$ & 510.7 & Sandy, silty clay & 1.63 & 2.7 & 1.08 \\
\hline $58 \mathrm{X}-2,12-15$ & 521.5 & Sandy silty clay and silty clay & 1.38 & 0.3 & 0.90 \\
\hline $59 \mathrm{X}-1,99-103$ & 530.5 & Homogeneous silty clay & 1.14 & 0.9 & 0.60 \\
\hline $60 X-2,110-115$ & 541.8 & Homogeneous silty clay & 0.85 & 0.3 & 0.17 \\
\hline $61 X-2,116-122$ & 551.5 & Homogeneous silty clay/claystone & 0.57 & 2.5 & 0.12 \\
\hline $62 \mathrm{X}-6,96-102$ & 566.9 & Homogeneous silty clay/claystone & 0.94 & 4.3 & 1.46 \\
\hline $63 \mathrm{X}-3,21-27$ & 571.0 & Homogeneous silty clay/claystone & 0.53 & 2.1 & 0.39 \\
\hline $64 X-3,118-124$ & 581.7 & Glauconitic sand/silt/clay, glauconitic silty clay, silty clay and clay & 1.97 & 6.8 & 2.00 \\
\hline $65 X-1,31-37$ & 587.4 & Moderately bioturbated clay and silty clay & 0.36 & 9.9 & 0.84 \\
\hline $67 \times-4,91-97$ & 611.7 & Moderately to heavily bioturbated silty clay and sandy silt & 0.68 & 2.7 & 1.47 \\
\hline $68 \times-2,91-95$ & 618.4 & Sand, clayey silt and silty clay & 1.57 & 7.2 & 1.90 \\
\hline $69 X-4,76-82$ & 630.8 & Clay & 0.40 & 7.5 & 0.24 \\
\hline $70 X-4,70-76$ & 640.4 & Slightly bioturbated clay and silty clay & 1.08 & 6.7 & 1.61 \\
\hline $71 X-1,26-31$ & 644.9 & Moderately bioturbated silty clay & 0.94 & 6.1 & 1.76 \\
\hline $72 X-5,20-25$ & 660.6 & Moderately bioturbated silty clay & 2.23 & 1.0 & 1.69 \\
\hline $73 \mathrm{X}-1,23-28$ & 664.3 & Homogeneous silty clay & 1.97 & 8.2 & 1.57 \\
\hline $74 X-4,120-126$ & 679.5 & Slightly bioturbated silty clay and clayey silt & 1.50 & 14.3 & 0.69 \\
\hline $75 X-6,26-32$ & 691.2 & Slightly to moderately bioturbated silty clay & 1.64 & 9.4 & 1.95 \\
\hline $76 X-5,20-26$ & 699.3 & Slightly to moderately bioturbated silty clay & 1.77 & 3.8 & 1.70 \\
\hline \multicolumn{6}{|l|}{$150-903 \mathrm{C}-$} \\
\hline $5 R-2,57-63$ & 507.6 & Glauconitic silty fine sand and glauconitic sandy silt & 1.68 & 3.6 & 0.70 \\
\hline $7 R-4,10-14$ & 519.9 & Sandy silt, glauconitic sandy silt and silty clay & 1.25 & 4.6 & 2.42 \\
\hline $9 \mathrm{R}-2,13-19$ & 588.1 & Slightly bioturbated clay & 0.54 & 3.6 & 0.25 \\
\hline $10 \mathrm{R}-1,110-116$ & 593.2 & Clay and glauconitic sandy clay & 0.61 & 2.7 & 0.19 \\
\hline $12 \mathrm{R}-1,89-94$ & 689.5 & Slightly to moderately bioturbated claystone & 1.63 & 5.2 & 1.89 \\
\hline $13 \mathrm{R}-1,95-101$ & 699.1 & Clayey silt to silty clay and glauconitic sandy silt & 1.16 & 3.8 & 1.31 \\
\hline $14 \mathrm{R}-4,27-33$ & 712.6 & Clayey silt and silty clay & 1.33 & 4.4 & 5.13 \\
\hline $15 R-4,24-30$ & 721.9 & Moderately to heavily bioturbated silty clay & 1.44 & 4.0 & 0.52 \\
\hline $16 \mathrm{R}-3,94-98$ & 730.9 & Silty clay and interbedded sandy silt and silty clay & 0.39 & 4.2 & 0.27 \\
\hline $17 \mathrm{R}-3,120-126$ & 741.0 & Claystone & 0.68 & 3.2 & 0.18 \\
\hline
\end{tabular}


Table 1 (continued).

\begin{tabular}{|c|c|c|c|c|c|}
\hline $\begin{array}{l}\text { Core, section, } \\
\text { interval }(\mathrm{cm})\end{array}$ & $\begin{array}{l}\text { Depth } \\
\text { (mbsf) }\end{array}$ & Lithology & $\begin{array}{l}\text { TOC } \\
\text { (wt\%) }\end{array}$ & $\begin{array}{l}\mathrm{CaCO}_{3} \\
(\mathrm{wt} \%)\end{array}$ & $\begin{array}{l}\text { Sulfur } \\
\text { (wt\%) }\end{array}$ \\
\hline $18 \mathrm{R}-3,75-78$ & 749.4 & Moderately to heavily bioturbated claystone & 0.73 & 4.3 & 0.27 \\
\hline $19 \mathrm{R}-2,86-89$ & 758.5 & Claystone and silty claystone & 1.82 & 5.8 & 0.82 \\
\hline $20 \mathrm{R}-5,100-103$ & 771.6 & Slightly to moderately bioturbated silty claystone & 1.95 & 5.0 & 1.71 \\
\hline $21 \mathrm{R}-1,37-42$ & 775.9 & Silty claystone and glauconite sandy siltstone & 1.85 & 3.9 & 1.73 \\
\hline $22 R-3,62-68$ & 788.8 & Silty claystone & 2.17 & 4.0 & 2.08 \\
\hline $23 \mathrm{R}-2,19-25$ & 796.6 & Moderately bioturbated silty claystone & 2.49 & 3.7 & 1.96 \\
\hline $24 \mathrm{R}-1,13-19$ & 804.2 & Moderately bioturbated silty claystone & 2.40 & 7.3 & 1.77 \\
\hline $25 \mathrm{R}-1,40-46$ & 814.2 & Moderately bioturbated silty claystone & 2.61 & 2.5 & 2.26 \\
\hline $27 \mathrm{R}-1,125-130$ & 834.3 & Moderately bioturbated silty claystone & 2.32 & 4.6 & 2.00 \\
\hline $28 \mathrm{R}-2,130-136$ & 845.5 & Slightly bioturbated silty claystone & 3.55 & 5.1 & 2.48 \\
\hline $29 \mathrm{R}-1,60-66$ & 853.0 & Moderately bioturbated silty claystone & 2.84 & 10.4 & 1.59 \\
\hline $31 \mathrm{R}-2,44-48$ & 873.6 & Silty claystone & 2.25 & 8.3 & 1.88 \\
\hline $32 \mathrm{R}-2,22-25$ & 883.1 & Silty claystone & 2.30 & 9.0 & 1.78 \\
\hline $33 \mathrm{R}-1,26-32$ & 891.3 & Homogeneous silty claystone & 3.21 & 11.7 & 1.38 \\
\hline $34 \mathrm{R}-1,50-55$ & 901.2 & Silty claystone and clauconitic silty claystone & 3.19 & 14.8 & 1.32 \\
\hline $35 \mathrm{R}-4,13-19$ & 913.9 & Moderately to heavily bioturbated silty claystone & 3.31 & 9.9 & 1.49 \\
\hline $36 \mathrm{R}-2,97-102$ & 922.4 & Slightly to moderately bioturbated silty claystone & 3.21 & 18.8 & 1.51 \\
\hline $37 \mathrm{R}-2,97-102$ & 932.2 & Silty claystone and dolomitic clayey siltstone & 2.81 & 10.3 & 1.69 \\
\hline $38 \mathrm{R}-1,60-65$ & 939.6 & Moderately bioturbated silty claystone & 2.55 & 16.1 & 1.87 \\
\hline $39 \mathrm{R}-3,60-63$ & 952.2 & Moderately to heavily bioturbated silty claystone & 3.01 & 21.8 & 1.20 \\
\hline $40 \mathrm{R}-2,100-106$ & 960.7 & Moderately bioturbated silty claystone & 2.81 & 7.3 & 1.55 \\
\hline $41 R-4,33-39$ & 972.3 & Silty claystone and glauconitic silty sandstone & 3.02 & 4.6 & 1.73 \\
\hline $44 R-2,60-64$ & 998.6 & Moderately bioturbated silty claystone & 2.45 & 25.4 & 1.37 \\
\hline $46 \mathrm{R}-1,99-105$ & 1007.2 & Moderately bioturbated silty claystone & 2.55 & 8.2 & 1.73 \\
\hline $47 R-2,62-66$ & 1027.6 & Slightly to moderately bioturbated silty claystone & 2.59 & 9.9 & 1.36 \\
\hline $48 \mathrm{R}-4,21-24$ & 1033.3 & Moderately bioturbated silty claystone & 3.14 & 23.5 & 1.24 \\
\hline $49 \mathrm{R}-4,144-147$ & 1039.7 & Slightly to moderately bioturbated silty claystone & 2.48 & 12.2 & 1.32 \\
\hline $51 R-4,35-41$ & 1057.9 & Clayey siltstone, silty claystone, glauconitic sandy siltstone and glauconitic silty sandstone & 2.55 & 13.7 & 1.15 \\
\hline $52 \mathrm{R}-3,6-11$ & 1067.2 & Heavily bioturbated nannofossil clayey chalk with foraminifer & 1.39 & 32.9 & 0.44 \\
\hline $53 \mathrm{R}-3,127-131$ & 1078.1 & Heavily bioturbated nannofossil clayey chalk with foraminifer & 0.50 & 40.5 & 0.23 \\
\hline $54 \mathrm{R}-3,4-10$ & 1086.4 & Heavily bioturbated nannofossil clayey chalk with foraminifer & 0.42 & 38.9 & 0.23 \\
\hline $55 \mathrm{R}-3,91-98$ & 1096.7 & Heavily bioturbated nannofossil clayey chalk with foraminifer & - & 33.4 & 0.23 \\
\hline $56 \mathrm{R}-2,89-92$ & 1104.0 & Slightly to heavily bioturbated nannofossil clayey chalk with foraminifera & 0.45 & 33.8 & 0.24 \\
\hline $57 \mathrm{R}-2,140-143$ & 1114.4 & Slightly to heavily bioturbated nannofossil clayey chalk with foraminifera & - & 43.5 & 0.24 \\
\hline $58 \mathrm{R}-6,36-41$ & 1127.6 & Heavily bioturbated porcellanitic nannofossil chalk & 0.77 & 53.5 & 0.07 \\
\hline $59 \mathrm{R}-2,62-69$ & 1131.6 & Heavily bioturbated calcareous porcellanite and porcellanitic nannofossil chalk & 0.26 & 42.0 & 0.05 \\
\hline $60 \mathrm{R}-2,30-34$ & 1140.6 & Heavily bioturbated calcareous porcellanite & 0.53 & 45.1 & 0.08 \\
\hline
\end{tabular}

Table 2. Lithology, organic carbon content, and elemental analysis data for core samples from Site $\mathbf{9 0 5}$.

\begin{tabular}{|c|c|c|c|c|c|}
\hline $\begin{array}{l}\text { Core, section, } \\
\text { interval }(\mathrm{cm})\end{array}$ & $\begin{array}{l}\text { Depth } \\
\text { (mbsf) }\end{array}$ & Lithology & $\begin{array}{c}\text { TOC } \\
(w t \%)\end{array}$ & $\begin{array}{l}\mathrm{CaCO}_{3} \\
(\mathrm{wt} \%)\end{array}$ & $\begin{array}{l}\text { Sulfur } \\
\text { (wt\%) }\end{array}$ \\
\hline \multicolumn{6}{|l|}{$150-905 \mathrm{~A}-$} \\
\hline $1 \mathrm{H}-2,55-60$ & 22.1 & Silty clay & 0.46 & 10.7 & 0.36 \\
\hline $2 \mathrm{H}-2,49-55$ & 31.5 & Silty clay & 0.35 & 8.8 & 0.51 \\
\hline $3 \mathrm{H}-4,22-28$ & 40.7 & Silty clay with laminated sandy clay & 0.33 & 6.0 & 0.22 \\
\hline $4 \mathrm{H}-1,100-106$ & 46.5 & Indistinctly bioturbated silty clay & 1.18 & 10.9 & 0.84 \\
\hline $5 \mathrm{H}-2,8-13$ & 56.6 & Silty clay and glauconite sand & 1.53 & 9.0 & 1.18 \\
\hline $7 \mathrm{H}-6,10-16$ & 70.6 & Slightly bioturbated silty clay & 1.92 & 1.8 & 1.60 \\
\hline $9 \mathrm{H}-3,30-36$ & 83.3 & Clay & 0.31 & 7.7 & 0.27 \\
\hline $10 \mathrm{H}-4,76-82$ & 94.2 & Clay & 0.35 & 8.1 & 0.35 \\
\hline $11 \mathrm{H}-2,132-138$ & 101.3 & Homogeneous silty clay & 0.27 & 12.8 & 0.16 \\
\hline $12 \mathrm{H}-1,115-120$ & 107.6 & Slightly sandy silty clay & 0.35 & 7.7 & 0.22 \\
\hline $13 \mathrm{H}-1,110-115$ & 112.6 & Silty clay & 0.32 & 8.1 & 0.24 \\
\hline $15 \mathrm{H}-2,120-126$ & 128.7 & Silty clay & 0.48 & 7.4 & 0.08 \\
\hline $16 \times-2,110-116$ & 137.1 & Clay & 0.37 & 8.2 & 0.19 \\
\hline $17 X-3,130-136$ & 148.4 & Homogeneous clay and silty clay & 0.39 & 10.0 & 0.14 \\
\hline $19 X-1,120-126$ & 164.6 & Silty clay & 0.34 & 7.6 & 0.30 \\
\hline $21 \mathrm{H}-3,10-16$ & 184.8 & Silty clay and sand & 0.31 & 7.9 & 0.23 \\
\hline $22 \mathrm{H}-1,54-66$ & 192.7 & Silty clay & 0.36 & 9.6 & 0.20 \\
\hline $23 \mathrm{H}-1,89-100$ & 196.6 & Silty clay & 0.42 & 8.6 & 0.18 \\
\hline $24 \mathrm{H}-1,86-91$ & 202.3 & Silty clay & 0.35 & 9.8 & 0.22 \\
\hline $25 \mathrm{H}-2,100-105$ & 213.5 & Homogeneous silty clay & 1.52 & 2.8 & 1.91 \\
\hline $26 \mathrm{H}-1,100-106$ & 216.0 & Homogeneous silty clay & 1.48 & 2.0 & 1.12 \\
\hline $27 \mathrm{X}-4,30-36$ & 223.8 & Homogeneous, moderately to heavily bioturbated silty clay & 1.19 & 11.7 & 1.31 \\
\hline $28 \mathrm{X}-2,90-95$ & 226.4 & Homogeneous, slightly bioturbated silty clay & 1.33 & 8.3 & 1.46 \\
\hline $29 X-2,130-136$ & 235.2 & Homogeneous, slightly bioturbated silty clay & 1.38 & 10.4 & 1.20 \\
\hline $30 \times-1,140-146$ & 243.4 & Homogeneous, slightly bioturbated silty clay & 1.36 & 10.8 & 1.48 \\
\hline $31 X-1,110-115$ & 252.8 & Homogeneous, slightly bioturbated silty clay & 1.40 & 9.1 & 1.41 \\
\hline $32 \times-2,110-116$ & 263.9 & Homogeneous, slightly bioturbated silty clay & 1.51 & 8.0 & 1.38 \\
\hline $33 \mathrm{X}-1,124-130$ & 272.2 & Homogeneous, rarely bioturbated silty clay & 1.09 & 15.7 & 0.72 \\
\hline $34 \mathrm{X}-1,14-20$ & 280.7 & Homogeneous, heavily bioturbated silty clay & 0.93 & 14.4 & 1.36 \\
\hline $35 X-4,130-136$ & 296.0 & Homogeneous, heavily bioturbated silty clay & 1.04 & 10.5 & 1.07 \\
\hline $36 X-2,110-116$ & 302.2 & Heavily bioturbated silty clay & 1.11 & 2.9 & 0.88 \\
\hline $37 X-2,110-122$ & 311.5 & Homogeneous silty clay & 0.89 & 12.1 & 0.69 \\
\hline $38 X-2,114-125$ & 320.9 & Homogeneous, slightly bioturbated silty clay & 1.18 & 8.8 & 0.96 \\
\hline $39 \times-4,27-33$ & 332.5 & Homogeneous, slightly to moderately bioturbated silty clay & 0.96 & 8.6 & 0.83 \\
\hline $40 X-3,60-67$ & 340.9 & Homogeneous, moderately bioturbated silty clay & 0.81 & 10.7 & 0.42 \\
\hline $41 X-3,19-25$ & 350.2 & Heavily bioturbated silty clay & 0.92 & 12.1 & 0.85 \\
\hline $42 X-2,36-41$ & 358.4 & Homogeneous, diatomaceous silty clay and clayey silt & 0.79 & 10.2 & 0.49 \\
\hline $43 X-3,90-96$ & 370.1 & Moderately bioturbated silty clay with foraminifera & 0.75 & 15.4 & 0.65 \\
\hline $44 X-6,40-46$ & 383.8 & Slightly to moderately bioturbated silty clay with foraminifera & 0.81 & 14.6 & 0.41 \\
\hline $45 X-3,37-42$ & 388.9 & Slightly bioturbated silty clay with foraminifera & 0.90 & 19.3 & 0.55 \\
\hline $46 X-3,15-21$ & 398.3 & Silty clay & 1.04 & 16.5 & 0.79 \\
\hline
\end{tabular}


Table 2 (continued).

\begin{tabular}{|c|c|c|c|c|c|}
\hline $\begin{array}{l}\text { Core, section, } \\
\text { interval }(\mathrm{cm})\end{array}$ & $\begin{array}{l}\text { Depth } \\
\text { (mbsf) }\end{array}$ & Lithology & $\begin{array}{c}\text { TOC } \\
\text { (wt \%) }\end{array}$ & $\begin{array}{l}\mathrm{CaCO}_{3} \\
(\mathrm{wt} \%)\end{array}$ & $\begin{array}{l}\text { Sulfur } \\
\text { (wt } \% \text { ) }\end{array}$ \\
\hline $47 X-4,140-146$ & 410.7 & Homogeneous, slightly bioturbated silty clay with foraminifera & 1.04 & 15.1 & 1.03 \\
\hline $48 \times-5,140-146$ & 421.9 & Homogeneous silty clay with foraminifera & 0.88 & 14.5 & 0.80 \\
\hline $49 \times-4,35-40$ & 428.6 & Slightly to rarely bioturbated silty clay with forminifera & 0.79 & 24.2 & 0.76 \\
\hline $50 X-1,10-16$ & 433.4 & Homogeneous silty clay & 0.71 & 11.8 & 0.48 \\
\hline $51 X-1,14-20$ & 443.1 & Slightly bioturbated silty clay & 0.76 & 19.7 & 0.39 \\
\hline $52 \times-6,120-126$ & 461.4 & Heavily bioturbated to homogenous silty clay & 0.83 & 17.2 & 0.48 \\
\hline $53 X-3,20-26$ & 465.5 & Moderately to heavily bioturbated silty clay & 0.91 & 8.5 & 0.49 \\
\hline $54 X-4,119-132$ & 476.6 & Homogeneous silty clay & 0.76 & 15.5 & 0.72 \\
\hline $55 \mathrm{X}-3,130-143$ & 485.9 & Silty clay, homgenized, scattered pyrite & 1.11 & 6.1 & 0.81 \\
\hline $56 \mathrm{X}-3,99-103$ & 495.3 & Slightly to moderately bioturbated silty clay & 1.31 & 3.5 & 0.99 \\
\hline $57 X-2,96-102$ & 503.1 & Homogeneous silty clay & 1.68 & 1.5 & 1.64 \\
\hline $58 \times-3,60-65$ & 513.8 & Heavily bioturbated to homogeneous clayey silt & 1.10 & 2.3 & 1.19 \\
\hline $59 \mathrm{X}-1,129-135$ & 521.2 & Moderately bioturbated clayey silt & 1.21 & 2.7 & 1.22 \\
\hline $60 X-6,20-25$ & 537.2 & Homogeneous, moderately bioturbated clayey silt and clayey silt/sand & 1.10 & 1.3 & 1.06 \\
\hline $61 X-1,31-36$ & 539.4 & Slightly to moderately bioturbated silty clay and sandy silty clay & 1.58 & 3.3 & 1,44 \\
\hline $62 X-1,20-26$ & 549.0 & Slightly to moderately bioturbated silty clay and conglomerate & 1.10 & 3.7 & 1.09 \\
\hline $63 X-5,139-146$ & 565.9 & Conglomerate and silty clay & 1.25 & 5.9 & 2.10 \\
\hline $64 X-1,5-10$ & 568.2 & Conglomerate, silty clay and carbonate-cemented siltstone & 1.68 & 5.1 & 1.79 \\
\hline $65 X-5,125-130$ & 585.0 & Homogeneous to slightly bioturbated silty clay & 0.76 & 5.7 & 1.40 \\
\hline $66 \mathrm{X}-1,17-22$ & 587.6 & Homogeneous to slightly bioturbated silty clay & 1.13 & 6.7 & 0.93 \\
\hline $68 X-4,103-108$ & 611.6 & Homogeneous to slightly bioturbated silty clay & 1.47 & 7.6 & 0.97 \\
\hline $69 X-5,15-21$ & 622.4 & Silty clay and mud-clast conglomerate & 1.45 & 2.2 & 1.41 \\
\hline $70 \times-4,22-28$ & 630.7 & Homogeneous to slightly bioturbated silty clay & 1.30 & 8.7 & 0.82 \\
\hline $71 X-3,119-125$ & 639.8 & Moderately to heavily bioturbated slightly silty clay & 0.99 & 11.1 & 1.23 \\
\hline $72 \times-4,15-24$ & 649.8 & Moderately bioturbated silty clay with diatoms and nannofossils & 0.97 & 17.4 & 0.62 \\
\hline
\end{tabular}

Table 3. Results of Rock-Eval pyrolysis for selected sediments from Site 903.

\begin{tabular}{|c|c|c|c|c|}
\hline $\begin{array}{l}\text { Core, section, } \\
\text { interval }(\mathrm{cm})\end{array}$ & $\begin{array}{l}\text { Depth } \\
\text { (mbsf) }\end{array}$ & $\begin{array}{c}\mathrm{HI} \\
(\mathrm{mgHC} / \mathrm{gTOC})\end{array}$ & $\begin{array}{c}\mathrm{OI} \\
\left(\mathrm{mgCO}_{2} / \mathrm{gTOC}\right)\end{array}$ & $\begin{array}{l}\mathrm{T}_{\max } \\
\left({ }^{\circ} \mathrm{C}\right)\end{array}$ \\
\hline \multicolumn{5}{|l|}{$150-903 \mathrm{~A}-$} \\
\hline $1 \mathrm{H}-4,94-100$ & 5.44 & 121 & 252 & 356 \\
\hline $13 \mathrm{H}-4,94-100$ & 119.37 & 38 & 270 & 446 \\
\hline $35 X-6,10-16$ & 305.50 & 129 & 172 & 416 \\
\hline $53 \times-4,90-96$ & 477.00 & 176 & 82 & 420 \\
\hline $55 \times-4,40-46$ & 495.80 & 184 & 66 & 420 \\
\hline $62 X-6,96-102$ & 566.96 & 329 & 146 & 424 \\
\hline $72 X-5,20-25$ & 660.60 & 240 & 100 & 423 \\
\hline \multicolumn{5}{|l|}{$150-903 \mathrm{C}$ - } \\
\hline $20 \mathrm{R}-5,100-103$ & 771.61 & 244 & 106 & 422 \\
\hline $34 \mathrm{R}-1,50-55$ & 901.20 & 289 & 81 & 417 \\
\hline $41 R-4,33-39$ & 972.33 & 253 & 77 & 413 \\
\hline $58 \mathrm{R}-6,36-41$ & 1127.62 & 23 & 229 & 413 \\
\hline
\end{tabular}

Note: $\mathrm{HI}=$ Hydrogen Index and $\mathrm{OI}=$ Oxygen Index.

the sediment samples from the upper slope of the New Jersey continental margin (477.0 mbsf, middle Miocene) is shown in Figure 7. The chromatogram demonstrates the abundance of long-chain alcohols of terrestrial origin that range from $\mathrm{C}_{17}$ to $\mathrm{C}_{32}$ and have a maximum at $\mathrm{C}_{28}$. They show a strong dominance of even carbon number homologs, and their absolute concentrations range up to $400 \mu \mathrm{g} / \mathrm{g}$ TOC. Also present is a complex mixture of steroidal alcohols, characteristic for a contribution of mainly marine organisms, with carbon skeletons from $\mathrm{C}_{27}$ to $\mathrm{C}_{30}$ and different positions of double bonds. Among the major constituents, $4 \alpha, 23,24$-trimethyl-5 $\alpha(\mathrm{H})$-cholest22 -en-3 $\beta$-ol (dinosterol) and 24-methylcholesta-5,22-dien-3 $\beta$-ol can be ascribed to an origin from dinoflagellates and diatoms, respectively (Volkman, 1986). The presence of 24-ethylcholest-5-en-3 $\beta$-ol and its saturated analog ( $\mathrm{k}$ and 1 in Fig. 7) is characteristic for organic matter from higher land plants but may also bear a (minor?) marine signature (Volkman, 1986).

The variations of absolute concentration with depth of three selected sterols are shown in Figure 8. There is not a steadily varying depth trend, but a tentative comparison (because of small sample number) with times of low sea level, as given by Miller and Mountain (1994), indicates that $4 \alpha, 23,24$-trimethyl-5 $\alpha(\mathrm{H})$-cholest-22-ene-3 $\beta$ ol has significantly lower concentrations at times of glaciation than in the other sediment sections. The opposite trend can be seen in the concentration of 24-ethylcholest-5-ene-3 $\beta$-ol, which may be characteristic for an increased supply of terrigenous organic matter by river transport to the outer continental shelf during times of low sea level.

\section{n-Fatty acids}

A distribution of $n$-fatty acids was observed, in all investigated samples, with a strong predominance of even carbon number homologs. Typical is a maximum at $\mathrm{C}_{16}$ for marine organic matter and at $C_{28}$ for terrigenous material (Cranwell, 1974; Simoneit, 1978). The occurrence of long-chain fatty acids $\left(>C_{22}\right)$ in most of the Site 903 samples reflects a continuous supply of remnants of higher land plants. A series of short-chain $\left(\mathrm{C}_{14}-\mathrm{C}_{18}\right)$ saturated and unsaturated fatty acids, which are presumably derived from marine algae, was recognized mainly in sediments of the middle and lower Miocene sections. Figure 9 shows the fatty acid distributions and absolute concentrations of individual compounds for two selected samples from the upper and the lower Miocene as examples of terrestrially and marine influenced fatty acid distributions, respectively.

\section{Ketones}

Long-chain di- and triunsaturated ketones were detected in each of the selected sediment samples from Site 903, and they were used to determine the alkenone unsaturation index $\left(\mathrm{U}^{\mathrm{k}}{ }_{37}\right)$ for reconstruction of paleo-sea-surface temperatures (Brassell, 1993; Prahl and Wakeham, 1987; Conte et al., 1992). The amounts of $\mathrm{C}_{37: 2}$ and $\mathrm{C}_{37: 3}$-alkenones in all samples are relatively small, ranging from 2 to $15 \mu \mathrm{g} / \mathrm{g}$ TOC. The paleo-sea-surface temperatures calculated from the $\mathrm{Uk}_{37}$ indices based on the calibration of Prahl and Wakeham (1987) are graphically represented in Figure 10. Other calibrations (e.g., Conte et al., 1992) lead only to small differences in absolute values which are not significant in view of the generally high temperature level throughout the entire sedimentary sequence. For the younger sediment sections, this high-temperature level of the oceanic surface water in the geological past is certainly caused by the proximity of the drilling location to the Gulf Stream, which transports warm surface water northwards along the east coast of the United States. For the older sediments, this may indicate that a similar oceanic surface current has existed even before the Middle American land mass separated the Atlantic Ocean completely from the Pacific Ocean.

General caution is necessary when using the alkenone ratios for determination of sea surface temperatures over longer periods of geological time, because $\mathrm{U}^{\mathrm{k}}{ }_{37}$ calibrations are based on the natural prod- 


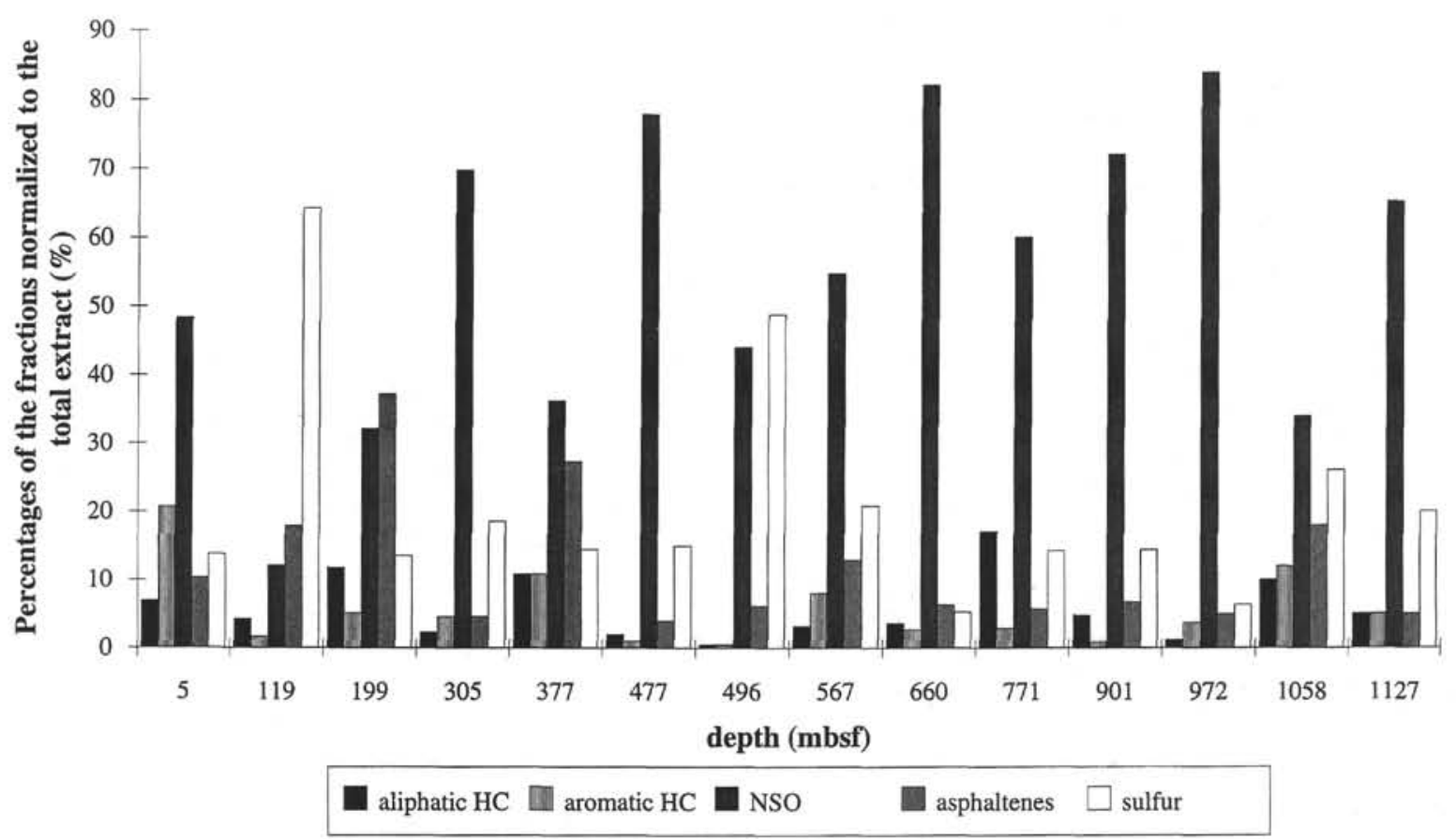

Figure 4. Histogram representation of gross extract fractions (including elemental sulfur) for 14 selected sediments from Site 903.

uct chemistry of the extant coccolithophore Emiliania huxleyi at different water temperatures. The fossil record of this organism dates back about $250 \mathrm{ka}$, which is only a short period compared with the 45-Ma age (middle Eocene) of the oldest sediments recovered on the New Jersey continental margin during Leg 150 . According to investigations of Marlowe et al. (1990), the morphological precursors of Emiliania huxleyi in the Gephyrocapsaceae family extend into the Eocene and were able to produce long-chain alkenones. There is no information for these organisms, however, on their adaptation of alkenone composition to growth temperature. Thus, the data provided here are completely based on the assumption of a conservative biochemistry of this family of organisms over many millions of years.

The drastic decrease of calculated water temperature to $3^{\circ} \mathrm{C}$ at a sediment depth of $1057 \mathrm{mbsf}$ is conspicuous. This temperature value is caused by the absence of the $\mathrm{C}_{37: 2}$ alkenone in the sample investigated. Possibly, this occurred by an unusual diagenetic alteration of the alkenone assemblage. Further investigations of adjacent samples will have to clarify this potential.

\section{CONCLUSIONS}

The sediments recovered during Leg 150 on the continental margin off New Jersey, spanning a range of $45 \mathrm{Ma}$, were obviously strongly influenced by a supply of terrigenous organic material. Variations in total organic carbon content are probably related to changes in the conditions for organic matter preservation in the sediments. This may be a consequence of fluctuations in the intensity of oxygenrich deep water currents at the continental margin over geological times. Relatively low hydrogen indices are consistent with the presence of (partly oxidized) terrestrial material and also confirm strong reworking of labile marine organic matter by sulfate-reducing and methanogenic bacteria, as indicated by the presence of up to $2 \%$ of sulfur and of abundant methane in the sediments.

Inspection of the aliphatic hydrocarbons corroborates the influence of terrigenous material caused by the dominance of higher plant wax alkanes. Elevated concentrations of sterenes and pentacyclic tri- terpenes were observed in sections where conditions were more favorable for preservation of labile marine organic matter (particularly in the middle and lower Miocene and in the Oligocene). The presence of hopanoids, mainly derived from bacteria, indicates a strong microbial reworking of the primary organic matter.

Molecular indicators for the presence of terrigenous organic matter were also found in the polar extract fractions (e.g., by the dominance of long-chain $n$-alcohols and $n$-fatty acids in many samples). Again, a carbon number shift to lower homologs indicates the preservation of higher relative amounts of marine organic matter. In the alcohol fraction, most steroids, including $4 \alpha, 23,24$-trimethyl-5 $\alpha(\mathrm{H})$ -

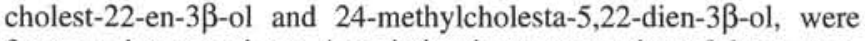
from marine organisms. A variation in concentration of these compounds with depth indicates changes in the supply of terrigenous organic matter, probably caused by sea-level fluctuations.

Paleo-sea-surface temperatures calculated from alkenone unsaturation indices showed a high temperature level off the present coast of New Jersey for the entire geological time span covered by the sedimentary section drilled during Leg 150 . This indicates the persistence of the Gulf Stream and an older analogous warm current in this region.

\section{ACKNOWLEDGMENTS}

We are grateful to Dr. Barbara Scholz-Böttcher (ICBM) for support in GC/MS analysis. We thank Dr. Richard W. Murray (Boston University) for his thorough review and helpful comments. Financial support was provided by the Deutsche Forschungsgemeinschaft (DFG, Bonn), Grant No. Ru 458/8.

\section{REFERENCES}

Amos, A.F., Gordon, A.L., and Schneider, E.D., 1971. Water masses and circulation patterns in the region of the Blake-Bahama Outer Ridge. DeepSea Res. Part A, 18:145-165.

Brassell, S.C., 1985. Molecular changes in sediment lipids as indicators of systematic early diagenesis. In Eglinton, G., Curtis, C.D., McKenzie, 
D.P., and Murchinson, D.G. (Eds.), Geochemistry of Buried Sediments. Philos. Trans. R. Soc. London A, 315:57-73.

1993. Applications of biomarkers for delineating marine paleoclimatic fluctuations during the Pleistocene. In Engel, M.H., and Macko, S.A. (Eds.), Organic Geochemistry: Principles and Applications: New York (Plenum), 699-738.

Bray, E.E., and Evans, E.D., 1961. Distribution of $n$-paraffins as a clue to recognition of source beds. Geochim. Cosmochim. Acta, 22:2-15.

Conte, M.H., Eglinton, G., and Madureira, L.A.S., 1992. Long-chain alkenones and alkyl alkenoates as palaeotemperature indicators: their production, flux and early sedimentary diagenesis in the eastern North Atlantic. Org. Geochem., 19:287-298.

Cranwell, P.A., 1974. Monocarboxylic acids in lake sediments, indicators, derived from terrestrial and aquatic biota, of paleoenvironmental trophic levels. Chem. Geol., 14:1-14.

Eglinton, G., and Calvin, M., 1967. Chemical fossils. Sci. Am., 216:32-43.

Espitalié, J., Laporte, J.L., Leplat, P., Madec, M., Marquis, F., Paulet, J., and Boutefeu, A., 1977. Méthode rapide de caractérisation des roches mères, de leur potentiel pétrolier et de leur degré d'évolution. Rev. Inst. Fr. Pet., 32:23-42.

Heezen, B.C., Hollister, C.D., and Ruddiman, W.F., 1966. Shaping of the continental rise by deep geostrophic contour currents. Science, 152:502508 .

Kolattukudy, P.E., 1976. Chemistry and Biochemistry of Natural Waxes: New York (Elsevier)

Marlowe, I.T., Brassell, S.C., Eglinton, G., and Green, J.C., 1990. Longchain alkenones and alkyl alkenoates and the fossil coccolith record of marine sediments. Chem. Geol., 88:349-375.

McCave, I.N., and Tucholke, B.E., 1986. Deep current-controlled sedimentation in the western North Atlantic. In Vogt, P.R., and Tucholke, B.E. (Eds.), The Western North Atlantic Region. Geol. Soc. Am., Geol. of North Am. Ser., M:451-468.

McEvoy, J., Eglinton, G., and Maxwell, J.R., 1981. Preliminary lipid analyses of sediments from Sections 467-3-3 and 467-97-2. In Yeats, R.S., Haq, B.U., et al., Init. Repts. DSDP, 63: Washington (U.S. Govt. Printing Office), 763-774.

Miller, K.G., and Mountain, G.S., 1994. Global sea-level change and the New Jersey margin. In Mountain, G.S., Miller, K.G., Blum, P., et al., Proc. ODP, Init. Repts., 150: College Station, TX (Ocean Drilling Program), 11-20.

Mountain, G.S., and Miller, K.G., 1993/94. Drilling for sea-level history on the New Jersey transect. Oceanus, 36:116-119.

Olsson, R.K., Melillo, A.J., and Schreiber, B.L., 1987. Miocene sea level events in the Maryland coastal plain and the offshore Baltimore Canyon trough. In Ross, C., and Haman, D. (Eds.), Timing and Depositional History of Eustatic Sequences: Constraints on Seismic Stratigraphy. Spec. Publ. Cushman Found. Foraminiferal Res., 24:85-97.
Olsson, R.K., and Wise, S.W., 1987. Upper Paleocene to middle Eocene depositional sequences and hiatuses in the New Jersey Atlantic Margin. In Ross, C., and Haman, D. (Eds.), Timing and Depositional History of Eustatic Sequences: Constraints on Seismic Stratigraphy. Spec. Publ. Cushman Found. Foraminiferal Res., 24:99-112.

Owens, J.P., and Gohn, G.S., 1985. Depositional history of the Cretaceous series in the U.S. coastal plain: stratigraphy, paleoenvironments, and tectonic controls of sedimentation. In Poag, C.W. (Ed.), Geologic Evolution of the United States Atlantic Margin: New York (Van Nostrand Reinhold), 25-86.

Philp, R.P., 1985. Biological markers in fossil fuel production. Mass Spectrom. Rev., 4:21-28.

Poynter, J., and Eglinton, G., 1990. Molecular composition of three sediments from Hole 717C: The Bengal Fan. In Cochran, J.R., Stow, D.A.V., et al., Proc. ODP, Sci. Results, 116: College Station, TX (Ocean Drilling Program), 155-161.

Prahl, F.G., and Wakeham, W.G., 1987. Calibration of unsaturation patterns in long-chain ketone compositions for paleotemperature assessment. Nature, 344:729-734.

Radke, M., Willsch, H., and Welte, D.H., 1980. Preparative hydrocarbon group type determination by automated medium pressure liquid chromatography. Anal. Chem., 52:406-411.

Shipboard Scientific Party, 1994a. Site 903. In Mountain, G.S., Miller, K.G., Blum, P., et al., Proc. ODP, Init. Repts., 150: College Station, TX (Ocean Drilling Program), 129-205.

, 1994b. Site 905. In Mountain, G.S., Miller, K.G., Blum, P., et al., Proc. ODP, Init. Repts., 150: College Station, TX (Ocean Drilling Program), 255-308.

Simoneit, B.R.T., 1978. The organic chemistry of marine sediments. In Riley, J.P., and Chester, R. (Eds.), Chemical Oceanography (2nd ed.) (Vol. 7): New York (Academic Press), 233-311.

Still, W.C., Kahn, M., and Mitra, A., 1978. Rapid chromatographic technique for preparative separations with moderate resolutions. J. Org. Chem., 43:2923-2926.

Volkman, J.K., 1986. A review of sterol markers for marine and terrigenous organic matter. Org. Geochem., 9:83-99.

Volkman, J.K., Allen, D.I., Stevenson, P.L., et al., 1986. Bacterial and algal hydrocarbons in sediments from a saline Antarctic lake, Ace Lake. In Leythaeuser, D., and Rullkötter, J. (Eds.), Advances in Organic Geochemistry 1985: Oxford (Pergamon Press), 671-681.

Date of initial receipt: 13 March 1995

Date of acceptance: 8 December 1995

Ms 150SR-040 

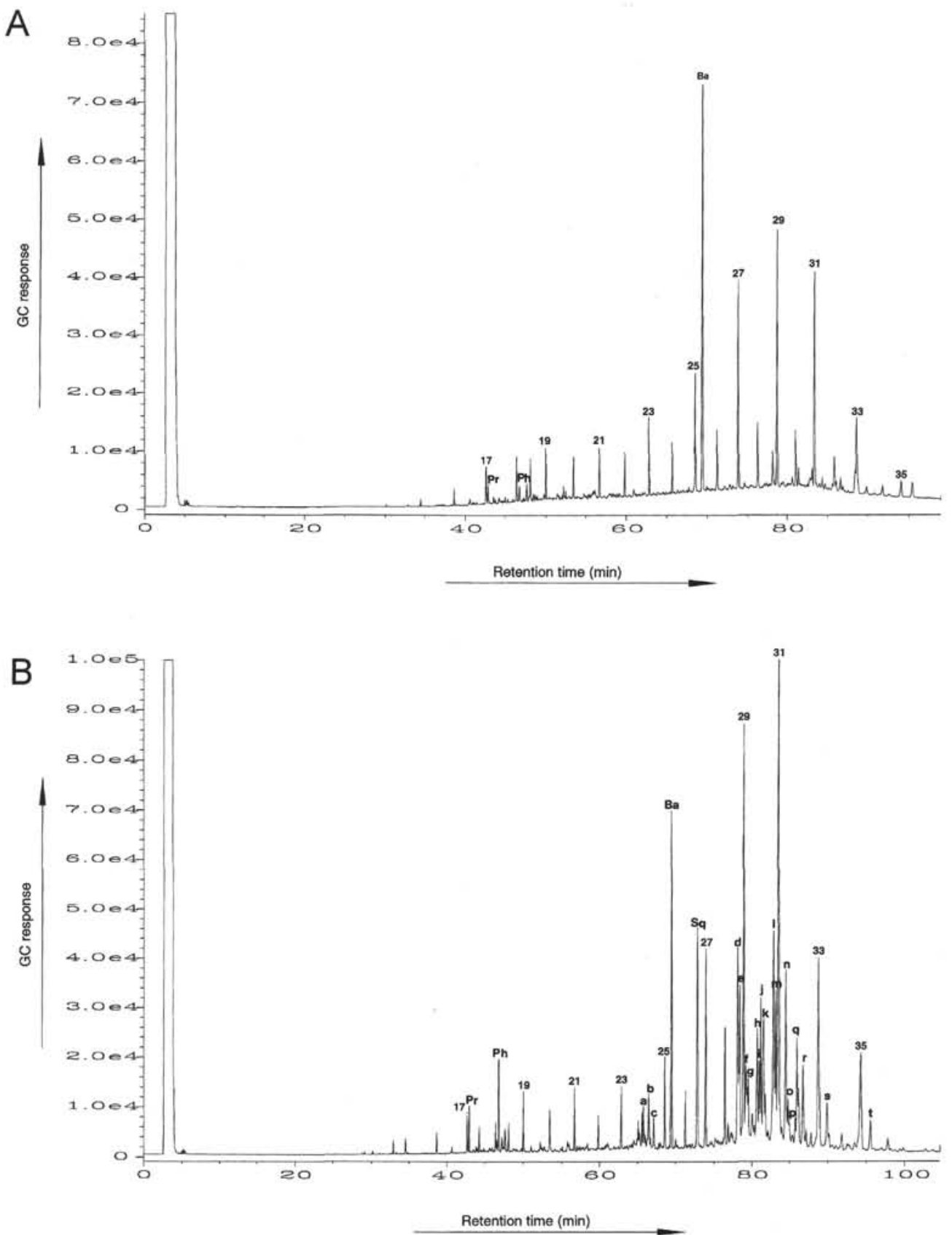

Figure 5. Capillary column gas chromatograms of the aliphatic hydrocarbon fractions in the extractable lipids of two selected sediments from Site 903 . Numbers indicate $n$-alkanes by their carbon chain length. See Table 4 for other labeled compounds. Ba (behenic acid methyl ester) and Sq (squalane) denote internal standards. A. Sample 150-903A-35X-6, 10-16 cm (305.50 mbsf, Pleistocene). B. Sample 150-903C-34R-1, 50-55 cm (901.20 mbsf, middle Miocene). 

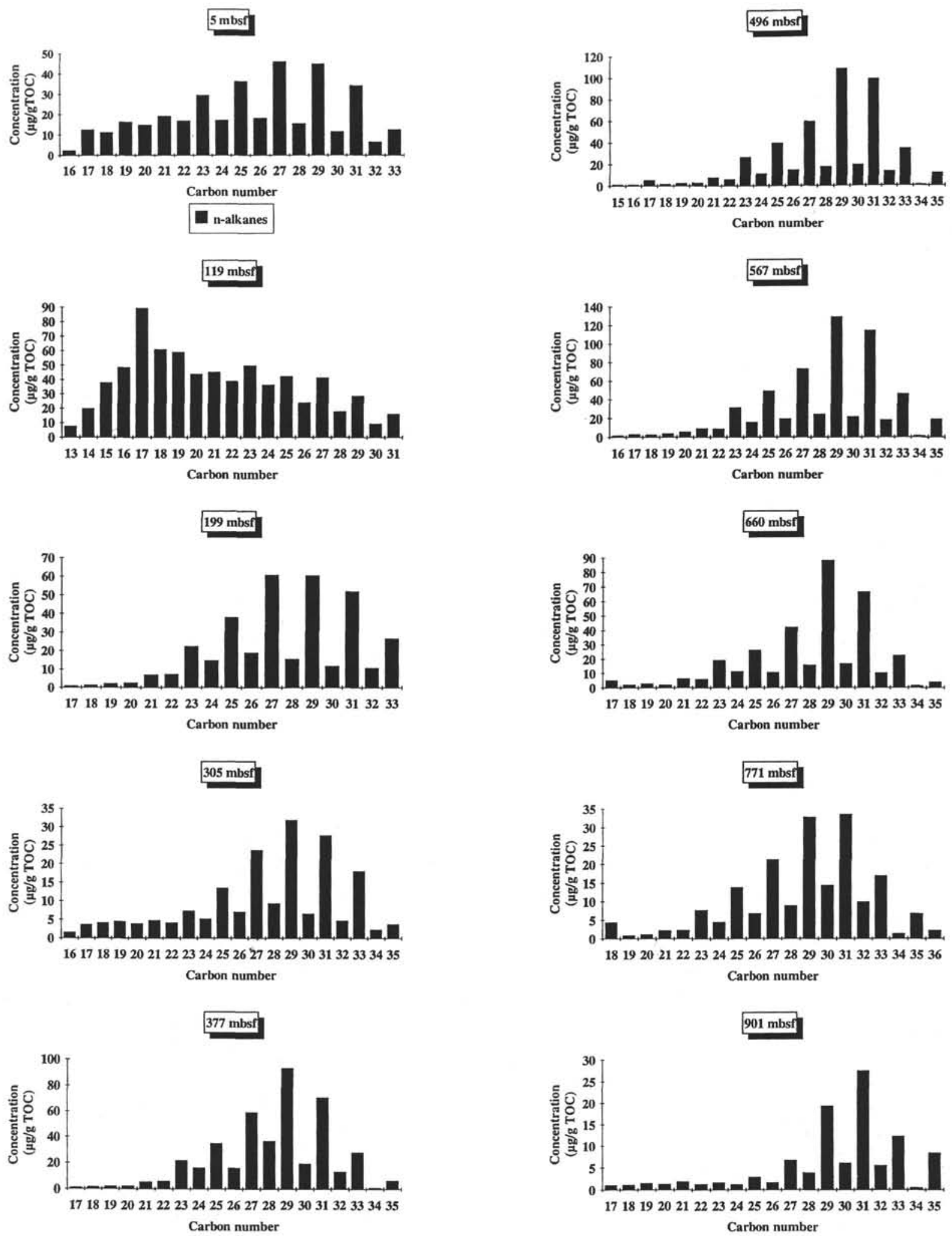

Figure 6. Carbon number distributions and absolute concentrations, normalized to TOC, of $n$-alkanes in lipid extracts of 14 selected sediments from Site 903. 
$477 \mathrm{mbst}$

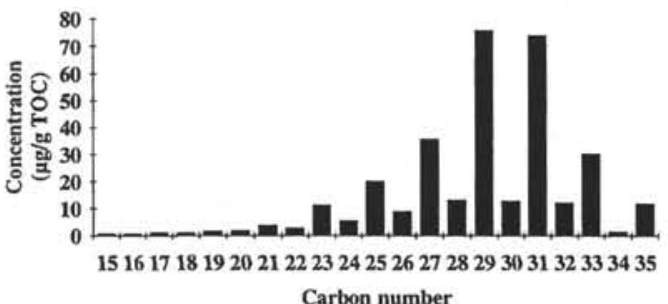

$1058 \mathrm{mbsf}$

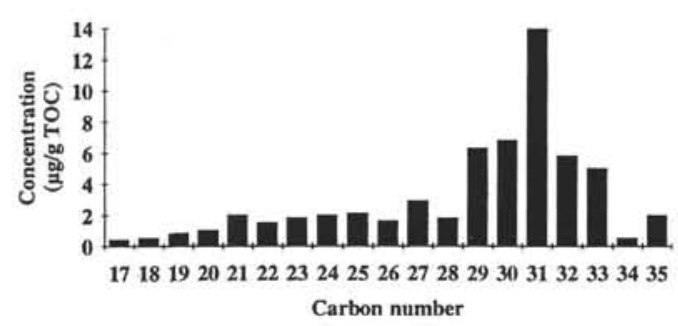

$972 \mathrm{mbsf}$
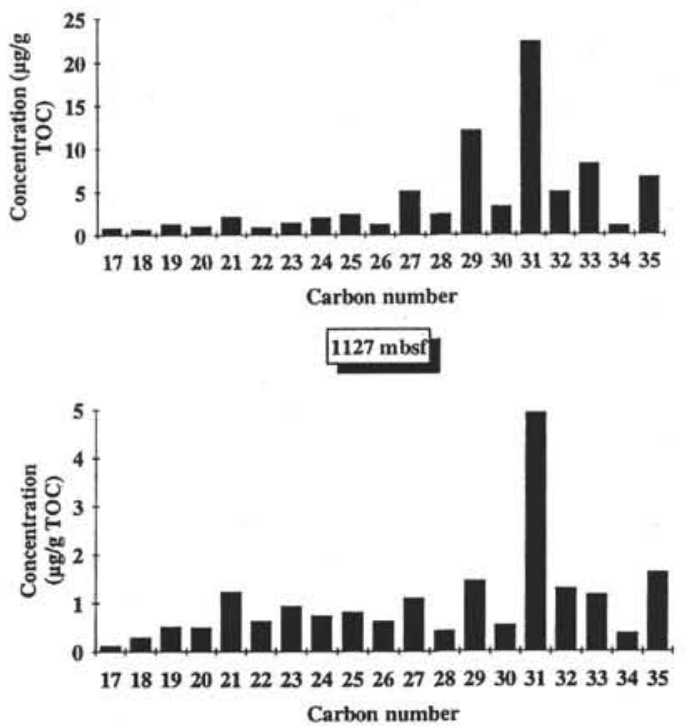

Figure 6 (continued).

Table 4. Compounds identified in the aliphatic hydrocarbon fractions of sediments from Site 903.

\begin{tabular}{|c|c|}
\hline Peak & Compound \\
\hline $\operatorname{Pr}$ & pristane \\
\hline $\mathrm{Ph}$ & phytane \\
\hline A & des-A-oleana-5(10),12-diene \\
\hline B & des-A-ursa-5(10),12-diene \\
\hline $\mathrm{C}$ & des-A-olean-12-ene \\
\hline D & cholest-4-ene \\
\hline E & cholest-5-ene \\
\hline $\mathrm{F}$ & 24-methylcholest-4-ene \\
\hline G & 24-methylcholest-5-ene \\
\hline $\mathrm{H}$ & 24-ethylcholest-4-ene \\
\hline I & 24-ethylcholest-2-ene \\
\hline J & 24-ethylcholest-5-ene \\
\hline $\mathrm{K}$ & 30-nor-hop-17(21)-ene \\
\hline $\mathrm{L}$ & hop-17(21)-ene \\
\hline M & 30 -nor-17ß $(\mathrm{H}), 21 \beta(\mathrm{H})$-hopane \\
\hline $\mathrm{N}$ & $17 \alpha(\mathrm{H})$-hopane \\
\hline $\mathrm{O}$ & fern-8-ene \\
\hline $\mathrm{P}$ & neo-hop-13(18)-ene \\
\hline$Q$ & 30-nor-moretane \\
\hline $\mathrm{R}$ & homo-hop-17(21)-ene \\
\hline $\mathrm{s}$ & $17 \beta(\mathrm{H}), 21 \beta(\mathrm{H})$-homo-hopane \\
\hline $\mathrm{T}$ & $17 \beta(\mathrm{H}), 21 \beta(\mathrm{H})$-dihomo-hopane \\
\hline
\end{tabular}

Table 5. Compounds identified in the alcohol fractions of Site 903 sediments.

\begin{tabular}{|c|c|}
\hline Peak & Compound \\
\hline $\mathrm{X}$ & phthalate (contaminant) \\
\hline $\mathrm{Ba}$ & behenic acid methylester (InjSTD) \\
\hline a & cholesta-5,22-dien-3 $\beta$-ol \\
\hline b & $5 \alpha(\mathrm{H})$-cholest- 22 -en- $3 \beta$-ol \\
\hline c & cholest-5-en-3ß-ol \\
\hline d & 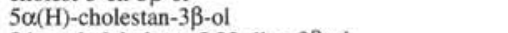 \\
\hline e & 24 -methylcholesta- 5,22 -dien-3 $\beta$-ol \\
\hline f & 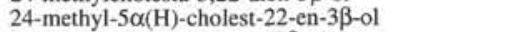 \\
\hline g & 4-methyl- $5 \alpha(\mathrm{H})$-cholestan-3 $\beta$-ol \\
\hline $\mathrm{h}$ & 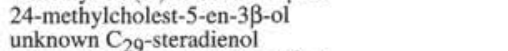 \\
\hline i & 24-ethylcholesta-5,22-dien-3ß-ol \\
\hline j & 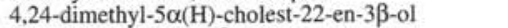 \\
\hline $\mathrm{k}$ & 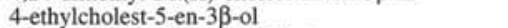 \\
\hline 1 & 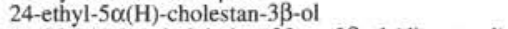 \\
\hline m & $4 \alpha, 23,24$-trimethylcholest-22-en-3 $\beta$-ol (dinosterol) \\
\hline $\mathrm{n}$ & $\beta$-amyrin \\
\hline
\end{tabular}




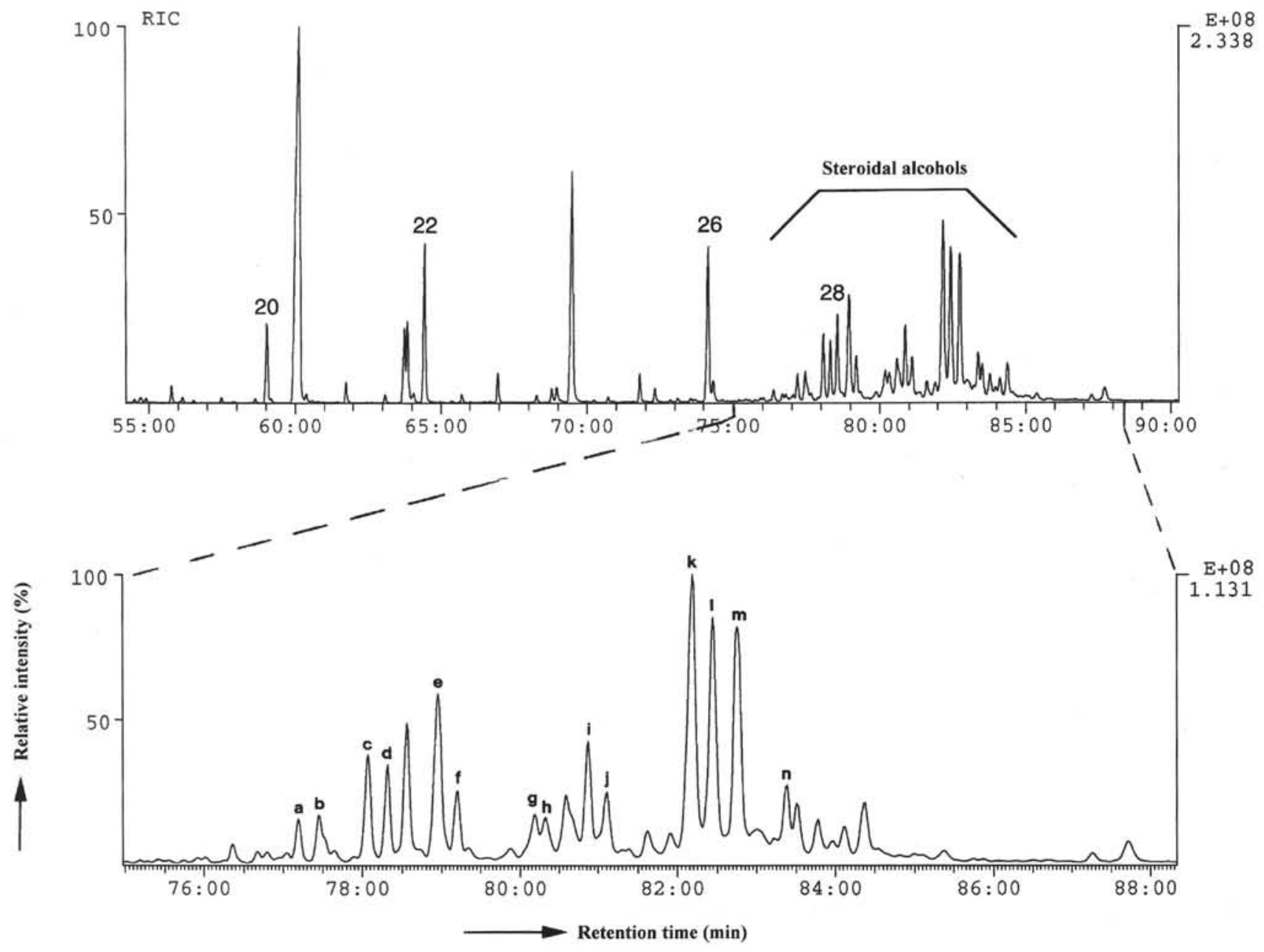

Figure 7. Partial reconstructed ion chromatogram of the alcohol fraction of the lipid extract of Sample 150-903A-53X-4, $90-96 \mathrm{~cm}$ (477.00 mbsf, upper Miocene) showing the sterol region. See Table 5 for compound identification. 


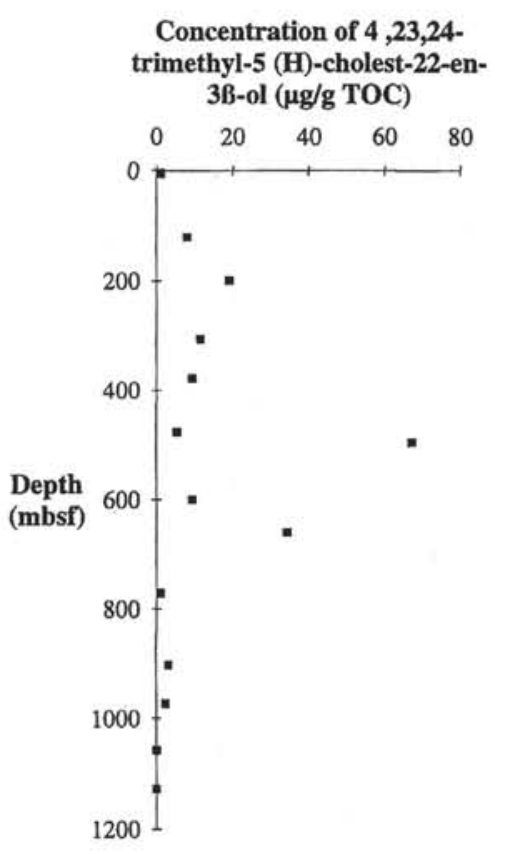

\section{Concentration of 24-methylcholesta- 5,22-dien-3B-ol ( $\mu \mathrm{g} / \mathrm{g}$ TOC)}

Concentration of 24-ethylcholest-5-en$3 B-o l(\mu g / g$ TOC)

Times of sea-level lowstand
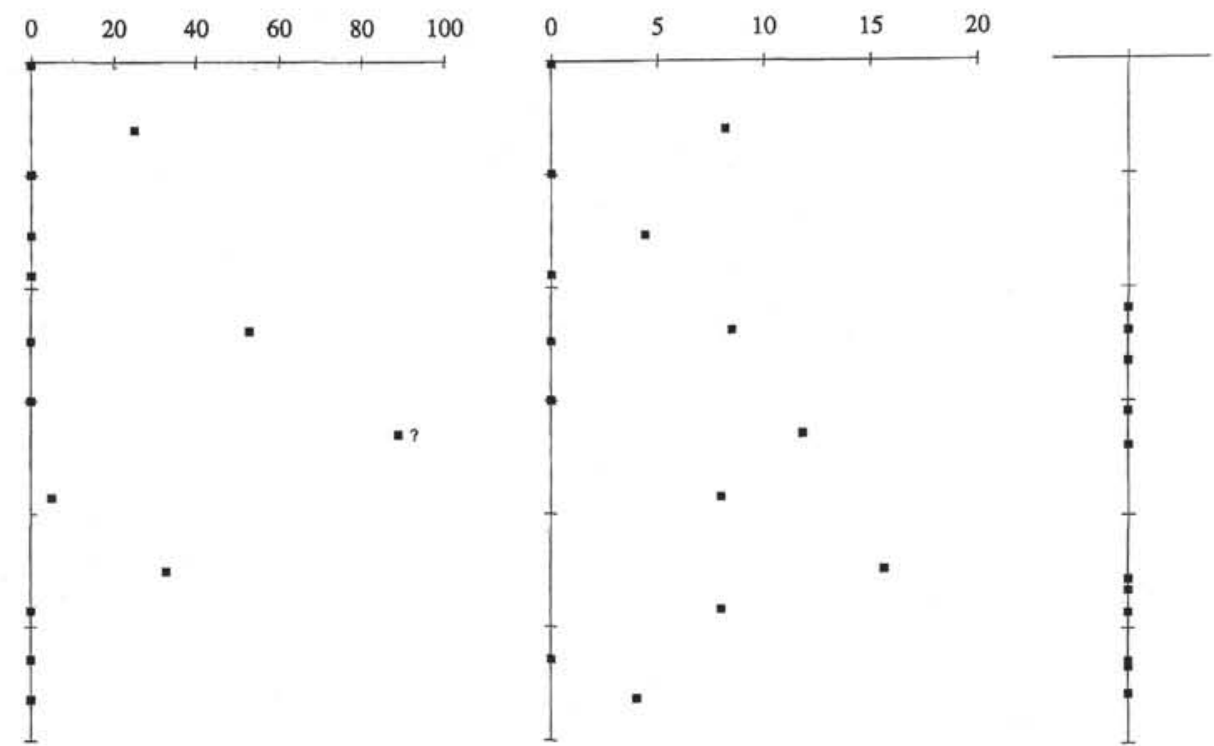

Figure 8. Downhole trends of absolute concentrations, normalized to TOC, for three selected steroid alcohols. Times of sea-level lowstand (Miller and Mountain, 1994) are indicated on the right.

A

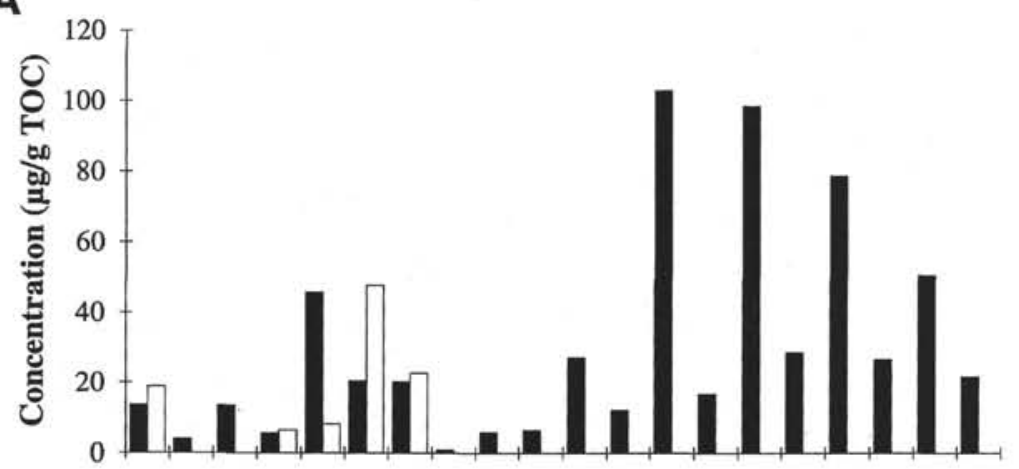

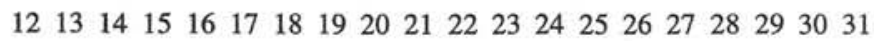

Carbon number

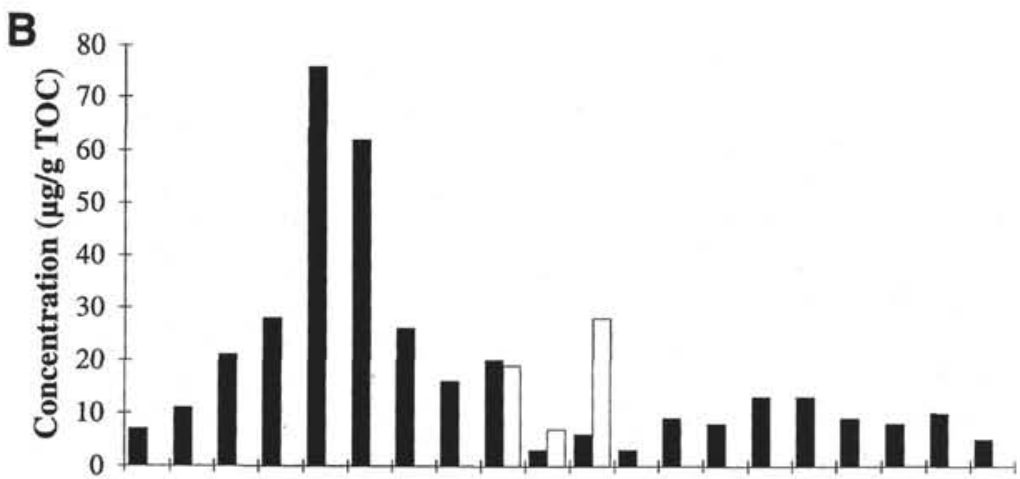

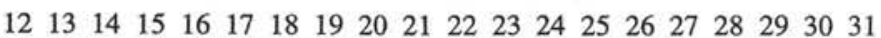

Carbon number

Figure 9. Carbon number distributions and absolute concentrations of saturated (closed bars) and monounsaturated fatty acids (open bars) in two selected sediments from Site 903. A. Sample 150-903A-55X-4, 40-46 cm (495.80 mbsf, upper Miocene). B. Sample 150-903C-41R-4, 33-39 cm (972.33 mbsf, lower Miocene). 


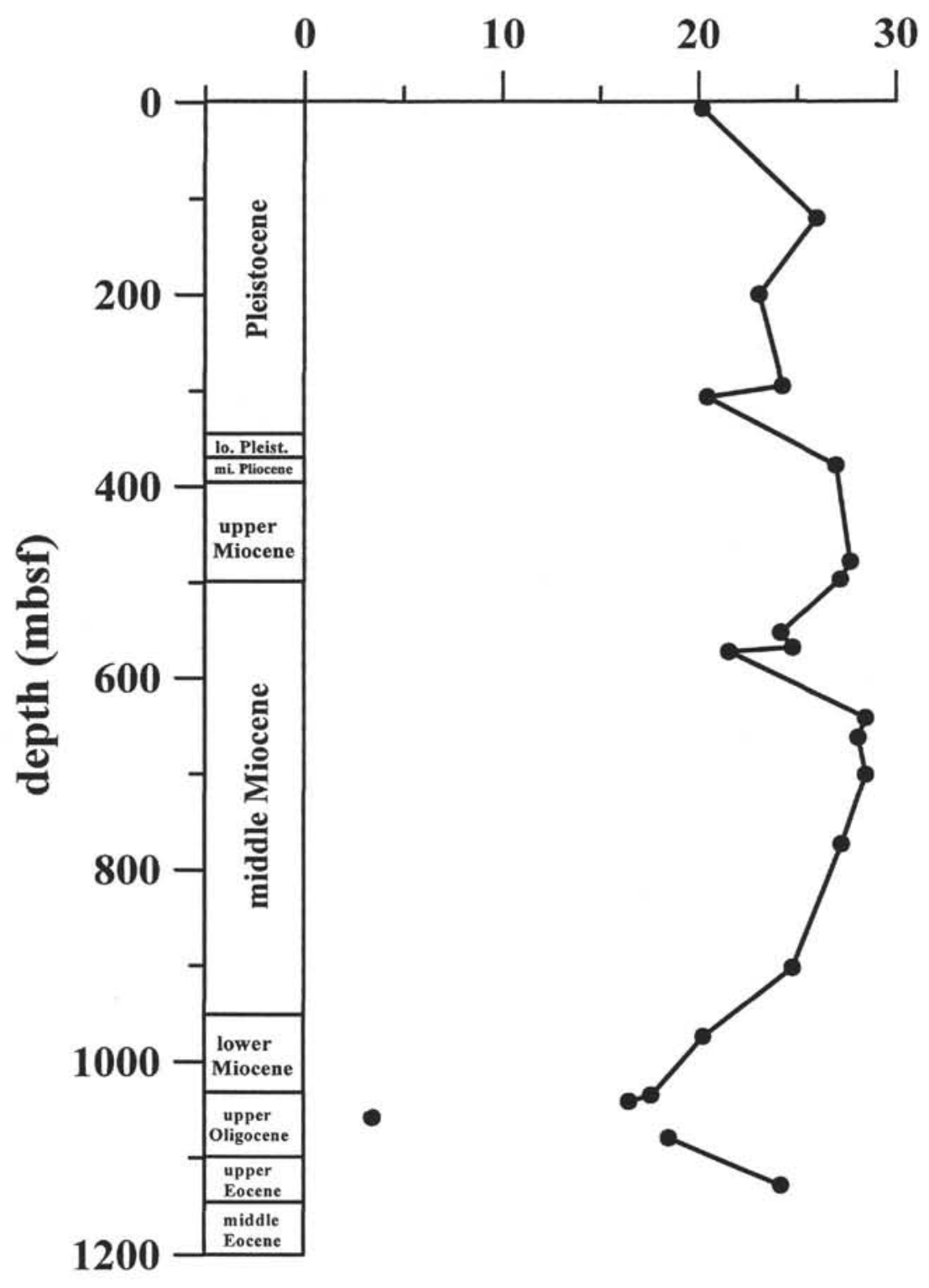

Figure 10. Paleo-sea-surface temperature on the New Jersey continental margin calculated from alkenone unsaturation data based on the calibration of Prahl and Wakeham (1987). 